AEI-2001-056

10 June 2001

\title{
Lorentzian 3d gravity with wormholes via matrix models
}

\author{
J. Ambjørn ${ }^{a}$, J. Jurkiewicz ${ }^{b}$, R. Loll ${ }^{\natural}$ and G. Vernizzi ${ }^{d}$ \\ ${ }^{a}$ The Niels Bohr Institute, \\ Blegdamsvej 17, DK-2100 Copenhagen Ø, Denmark \\ email: ambjorn@nbi.dk \\ ${ }^{b}$ Marian Smoluchowski Institute of Physics, Jagellonian University, \\ Reymonta 4, PL 30-059 Krakow, Poland \\ email: jjurkiew@th.if.uj.edu.pl \\ ${ }^{c}$ Albert-Einstein-Institut, \\ Max-Planck-Institut für Gravitationsphysik, \\ Am Mühlenberg 1, D-14476 Golm, Germany \\ email:loll@aei-potsdam.mpg.de \\ ${ }^{d}$ Department of Theoretical Physics, Oxford University \\ 1 Keble Road, Oxford, OX1 3NP, United Kingdom \\ email:vernizzi@thphys.ox.ac.uk
}

\begin{abstract}
We uncover a surprising correspondence between a non-perturbative formulation of three-dimensional Lorentzian quantum gravity and a hermitian two-matrix model with ABAB-interaction. The gravitational transfer matrix can be expressed as the logarithm of a two-matrix integral, and we deduce from the known structure of the latter that the model has two phases. In the phase of weak gravity, well-defined two-dimensional universes propagate in proper time, whereas in the strong-coupling phase the spatial hypersurfaces disintegrate into many components connected by wormholes.
\end{abstract}

\footnotetext{
${ }^{1}$ address from Sept '01: Institute for Theoretical Physics, Utrecht University, Minnaertgebouw, Leuvenlaan 4, NL-3584 CE Utrecht
} 


\section{Introduction}

A major task of modern theoretical physics is to unite quantum mechanics with the theory of gravity and to understand "quantum geometry". In four dimensions, this is proving a difficult task and there is no general consensus on which direction to take. The so-called "string community", originating from quantum field theory, claims that M-theory provides the only viable road to unifying quantum mechanics and gravity, while people coming from the theory of relativity tend to favour approaches based on canonical quantization. So far neither of these approaches has given us a detailed understanding of the microscopic quantum geometry of the real space-time.

The situation is considerably better in dimension $d<4$. Although lowerdimensional models do not possess propagating gravitational degrees of freedom, their geometries are still subject to quantum fluctuations, and the quantum theories are non-trivial. One may use conventional quantum field-theoretic methods to investigate the coupling between matter and gravity, and to define and calculate diffeomorphism-invariant correlation functions and the dynamically generated fractal dimension of quantum space-time. Of course, we have no way of knowing how relevant the study of the lower-dimensional theories will be for the eventual theory of four-dimensional quantum gravity, but it is clear that there are structural similarities and certainly some of the same questions can be asked.

A detailed and explicit analysis exists in two space-time dimensions. One can calculate the anomalous dimensions acquired by matter fields when coupled to $2 \mathrm{~d}$ Euclidean gravity [1, 2]. It is also understood that a typical two-dimensional Euclidean geometry contributing to the gravitational path integral has a fractal structure with Hausdorff dimension four, much in the same way as a typical path in the path integral for a particle is also fractal, with Hausdorff dimension two.

The fractal structure is best understood by introducing a geodesic "time" on the two-geometries. Euclidean $2 \mathrm{~d}$ gravity is characterized by the fact that an infinite number of baby universes branches off the one-dimensional spatial slice as it evolves in this "time" [3, 4, 5]. While such a process is unavoidable in two-dimensional gravity models coming from string theories (describing non-critical strings), there is nothing in a theory of quantum gravity which demands that space should be allowed to split into disconnected parts. In the context of canonical gravity this would require a "third quantization" to enable the destruction and creation of baby universes, a possibility that is not usually considered.

Not allowing for the creation of baby universes leads to a new two-dimensional quantum theory, called Lorentzian quantum gravity. The name derives from the fact that the sum over geometries in the path integral includes only a particular subclass of Euclidean geometries which are obtained through a Wick rotation from a set of Lorentzian space-times with a well-defined causal and globally hyperbolic structure [6]. Interestingly, the resulting quantum theory is different from the Euclidean one. For instance, the fractal dimension of a typical geometry is two and not four, and the coupling of matter and geometry creates no anomalous scaling dimensions for 
the matter fields [0, 8]. The detailed relation between the geometries of the two models is well understood [9].

Very important in the study of two-dimensional quantum gravity have been methods and concepts from statistical physics [10, 5]. The regularization in both the Euclidean and Lorentzian case uses so-called dynamical triangulations. In this approach, geometries are created by gluing together large numbers of identical triangular building blocks. The geodesic edge length of the triangles is a measure of the fineness of the simplicial lattice and defines a diffeomorphism-invariant cutoff of the theory. The action of such a piecewise linear two-geometry is calculated by Regge's prescription, and the state sum over geometries can in many cases be performed explicitly. The scaling limit (i.e. taking the number of triangles to infinity), defines the continuum limit of these models.

Since in both Euclidean and Lorentzian $2 d$ dynamical triangulations we have a concept of time ("geodesic" time and "proper" time respectively), a transfer matrix can be introduced which describes the evolution (the transition amplitude) between the spatial configurations at time $t$ and $t+a$, where $a$ is a discrete lattice spacing. The notion of a transfer matrix is familiar from quantum field theories on fixed lattices. It allows us to extract the continuum Hamiltonian of the system in the limit as $a \rightarrow 0$ according to

$$
\left\langle\phi(x)|\hat{T}| \phi^{\prime}\left(x^{\prime}\right)\right\rangle=\left\langle\phi(x)\left|\mathrm{e}^{-a \hat{H}}\right| \phi^{\prime}\left(x^{\prime}\right)\right\rangle \rightarrow\left\langle\phi(x)\left|\left(\hat{1}-a \hat{H}+O\left(a^{2}\right)\right)\right| \phi^{\prime}\left(x^{\prime}\right)\right\rangle .
$$

This strategy has been applied successfully in Euclidean and Lorentzian two-dimensional quantum gravity.

While all of this works beautifully in dimension two, where it has given us a number of powerful analytical tools, the situation is quite different when one tries to use the method of dynamical triangulations to obtain a theory of Euclidean quantum gravity in higher dimensions. Such models have been investigated mainly through numerical simulations [11, 12, and the results have so far been disappointing: no interesting continuum limits seem to exist [13]. 20 In fact, this was one of the main motivations for constructing alternative Lorentzian models of dynamical triangulations, which we have already mentioned in the two-dimensional context. They have recently been shown to exist as well-defined regularized models of quantum gravity also in three and four space-time dimensions [15, 16] (see also [17] for a review of discrete Lorentzian gravity).

An investigation of the continuum properties of the three-dimensional Lorentzian gravity model has already begun. Computer simulations show that it avoids some of the problems of Euclidean simplicial quantum gravity and most likely has a continuum limit [18], thus fulfilling some of the hopes raised by the two-dimensional Lorentzian model. As is well-known from previous attempts, analytic tools are hard to come by in statistical models of quantum geometry in $d>2$. In particular,

\footnotetext{
${ }^{2}$ This seems to be part of a general pattern, since also other non-perturbative discrete approaches to four-dimensional Euclidean quantum gravity have had little success, see [14] for a review.
} 
the matrix-model methods that proved so powerful in two dimensions have not yet been made into a useful calculational tool in higher-dimensional quantum gravity. The purpose of this article is to demonstrate that in three-dimensional Lorentzian quantum gravity, such analytic matrix-model techniques can indeed be employed.

The remainder of this article is organized as follows. In Sec. 2 we describe the quantum gravity model in terms of simplicial geometries and define its transfer matrix. At the discretized level, it is a simple variant of the three-dimensional Lorentzian model introduced in [15, 16] and studied by Monte Carlo simulations in [18, [1]. Motivated by some well-known properties of $(2+1)$-dimensional quantum gravity, we perform an integration over all but one of the spatial geometric degrees of freedom in Sec. 3. In the following Sec. 4 we remind the reader of how to obtain the continuum Hamiltonian from the resulting transfer matrix. The correspondence of $3 \mathrm{~d}$ Lorentzian quantum gravity with the already partially solved two-matrix model [20] with $A B A B$-interaction is established in Sec. 2. We reinterpret the phase structure of this matrix model in terms of geometry in Sec. 6 . This also involves a discussion of other, closely related matrix models with so-called touching-interactions. We briefly comment on the status of the full and as yet unsolved $A B A B$-matrix model in Sec. 77. In Sec. 8 we describe how taking the continuum limit in the $A B A B$-model fits in with our previous considerations of this limit in three-dimensional gravity. We end with an outlook in Sec. 9. The appendix contains a derivation of the gravitational action in terms of the $3 \mathrm{~d}$ building blocks used in this article.

\section{Lorentzian 3d gravity from pyramids and tetra- hedra}

The motivation for constructing non-perturbative gravitational path integrals of Lorentzian geometries and the general properties of the dynamically triangulated model in three dimensions were described in [15, 16]. For our present purposes it is convenient to consider a slightly modified regularization which can be related to a quartic matrix model. (The cubic matrix model that would correspond to our original model which uses only tetrahedra has not yet been solved.)

In the regularized model, (proper) time $t$ is discretized into integer lattice steps of unit one. The spatial slices at $t=0,1, \ldots$ are piecewise linear manifolds of spherical topology, constructed by gluing together flat squares with edge length $l_{s}=a$ (rather than the equilateral triangles with edge length $l_{s}=a$ of references [15, 16, 18]).

The geometry of a spatial slice is uniquely fixed by this length assignment and by its connectivity matrix, specifying which pairs of squares are glued together along a common edge. Two spatial slices at $t$ and $t+1$ form the space-like boundaries of a three-dimensional piecewise-linear manifold "sandwich" that lies in between the slices and has topology $S^{2} \times[0,1]$. The fundamental three-dimensional building blocks used for "filling in" are regular pyramids with square base and tetrahedra 


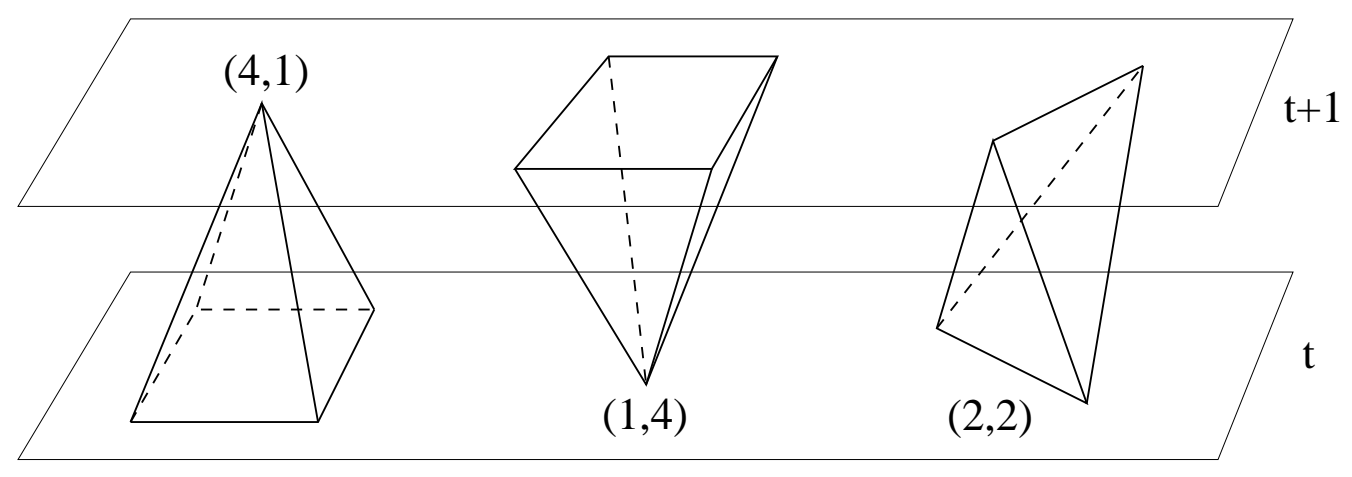

Figure 1: Pyramids and tetrahedra can be used to discretize 3d Lorentzian spacetimes. We show the three types of fundamental building blocks and their location with respect to the spatial hypersurfaces of constant integer- $t$.

(see Fig. 11). The pyramids have either their base in the $t$-plane and their tip in the $t+1$-plane - in which case we call them $(4,1)$-pyramids - or vice versa for the $(1,4)$-pyramids. In addition, each pyramid has four time-like edges of equal squared length $l_{t}^{2}$ connecting the neighbouring slices ${ }^{3}$. Two pyramids of equal orientation can be glued together along a time-like triangle. Since (4,1)-pyramids with base at $t$ and $(1,4)$-pyramids with base at $t+1$ cannot share a triangular face, we need an additional type of building block, namely, a (2,2)-tetrahedron with one space-like edge (of length $l_{s}$ ) each in the $t$ - and $t+1$-planes (Fig. 1). Its remaining four time-like edges have again squared length $l_{t}^{2}$, so that its faces can be glued to the pyramids of both types.

We will in the following use the term "quadrangulation" to denote a piecewiseflat geometry made of $(4,1)$-, $(1,4)$ - and $(2,2)$-building blocks, as well as their twodimensional spatial sections. (Note that a $3 \mathrm{~d}$ quadrangulation may be thought of as a particular kind of a three-dimensional triangulation, obtained by cutting all pyramids into pairs of tetrahedra.) Starting from a $S^{2}$-quadrangulation at time $t=0$, we can by successive gluing build up a three-dimensional space-time of length $t$ in the time direction, consisting of $t+1$ spatial spheres, and $t$ "sandwiches" in between.

Although our choice of allowed discretized space-times is of course motivated by the causal structure associated with the physical Lorentzian signature, we will from now on do all calculations for the already Wick-rotated Euclidean geometries. Without loss of generality, we set $l_{t}^{2}=\beta l_{s}^{2} \equiv \beta a^{2}$, for $\beta>1 / 2$ (because of triangle inequalities). Next, we must compute the Boltzmann weight $\mathrm{e}^{-\mathrm{S}}$ associated with a given three-geometry. The Euclidean Einstein action in the continuum is given by

$$
S^{\text {cont }}=-\frac{1}{16 \pi G_{N}} \int_{M} \mathrm{~d}^{3} x \sqrt{g(x)}(R(x)-2 \Lambda)-\frac{1}{8 \pi G_{N}} \int_{\partial M} \mathrm{~d}^{2} x \sqrt{h(x)} K(x),
$$

\footnotetext{
${ }^{3}$ Our language here is "Lorentzian" in line with our general philosophy [15, 16], where a Wick rotation corresponds to a sign flip of $l_{t}^{2}$.
} 
where $h(x)$ is the induced metric and $K(x)$ the trace of the extrinsic curvature on the boundary $\partial M$ of the manifold $M$, and $G_{N}$ and $\Lambda$ are the gravitational and cosmological coupling constants. As usual for 3d piecewise linear manifolds, the curvature is concentrated on the one-dimensional edges or links, and proportional to the deficit angle under rotation around a link in a plane perpendicular to it. Using the standard Regge prescription for computing the total scalar curvature of a simplicial manifold, the discrete counterpart of (2) is derived in the appendix, where we also discuss the inverse Wick rotation of the action.

Let us adopt the following notation: spatial quadrangulations at integer- $t$ are called $\mathcal{T}(t)$ and a sandwich geometry $\mathcal{G}(\mathcal{T}(t), \mathcal{T}(t+1))$. One piece of information contained in the data characterizing the geometry $\mathcal{G}(\mathcal{T}(t), \mathcal{T}(t+1))$ in the interval $[t, t+1]$ is the set of three numbers $N_{41}(t), N_{14}(t)$ and $N_{22}(t)$ of building blocks of the three types. For a given geometry $\mathcal{G}$, the action depends only on these three bulk variables,

$S[\mathcal{G}(\mathcal{T}(t), \mathcal{T}(t+1))]=c_{0}-\tilde{k}\left(N_{41}(t)+N_{14}(t)-N_{22}(t)\right)+\tilde{\lambda}\left(N_{41}(t)+N_{14}(t)+\frac{1}{2} N_{22}(t)\right)$.

In (3), $N_{41}(t)+N_{14}(t)-N_{22}(t)$ is proportional to the integrated scalar curvature (including the extrinsic curvature terms for the boundaries) between $t$ and $t+1$, while $N_{41}(t)+N_{14}(t)+\frac{1}{2} N_{22}(t)$ is proportional to the three-volume of this piece of spacetime. The dimensionless coupling constants $\tilde{k}$ and $\tilde{\lambda}$ are proportional to the bare inverse gravitational coupling constant $1 / G_{N}$ and the bare cosmological coupling constant $\Lambda$. The explicit form of the coupling constants as functions of $\beta, G_{N}$ and $\Lambda$ can be found in the appendix.

When we start stacking up sandwich geometries, the action (3) is by construction additive, so that the total action for a space-time extending $t$ steps in the timedirection becomes

$$
S[\tilde{k}, \tilde{\lambda}]=c_{0} t-\tilde{k}\left(N_{41}+N_{14}-N_{22}\right)+\tilde{\lambda}\left(N_{41}+N_{14}+\frac{1}{2} N_{22}\right)
$$

where $N_{41}, N_{14}$ and $N_{22}$ denote now the total numbers of $(4,1)$-, $(1,4)$ - and $(2,2)$ building blocks.

We can now define the transfer matrix associated with a unit step of proper time ast

$$
\langle\mathcal{T}(t+1)|\hat{T}| \mathcal{T}(t)\rangle=\sum_{\mathcal{G}(\mathcal{T}(t), \mathcal{T}(t+1))} \frac{1}{C_{\mathcal{G}}} \mathrm{e}^{-S[\mathcal{G}(\mathcal{T}(t), \mathcal{T}(t+1))]},
$$

${ }^{4}$ Following [16], we introduce quantum states $|\mathcal{T}\rangle$ at fixed $t$, labelled by inequivalent spatial geometries and normalized according to

$$
\left\langle\mathcal{T}_{1} \mid \mathcal{T}_{2}\right\rangle=\frac{1}{C_{\mathcal{T}_{1}}} \delta_{\mathcal{T}_{1}, \mathcal{T}_{2}}, \quad \sum_{\mathcal{T}} C_{\mathcal{T}}|\mathcal{T}\rangle\langle\mathcal{T}|=\hat{1},
$$

where $C_{\mathcal{T}}$ is the symmetry factor of the quadrangulation $\mathcal{T}$. 
where the summation is over all distinct three-geometries $\mathcal{G}(\mathcal{T}(t), \mathcal{T}(t+1))$ whose boundary geometries are $\mathcal{T}(t)$ and $\mathcal{T}(t+1)$. $C_{\mathcal{G}}$ denotes the symmetry factor of the quadrangulation $\mathcal{G}$, i.e. the order of the automorphism group of $\mathcal{G}$.

Let $\mathcal{T}_{1}=\mathcal{T}(t)$ contain $N_{41}(\mathcal{T}(t))$ squares and $\mathcal{T}_{2}=\mathcal{T}(t+1)$ contain $N_{14}(\mathcal{T}(t+1))$ squares. Substituting the sandwich action (3) into (5), we can write

$$
\left\langle\mathcal{T}_{2}|\hat{T}| \mathcal{T}_{1}\right\rangle=\mathrm{e}^{-c_{0}} \mathrm{e}^{-(\tilde{\lambda}-\tilde{k})\left(N_{41}\left(\mathcal{T}_{1}\right)+N_{14}\left(\mathcal{T}_{2}\right)\right)} \sum_{N_{22}=N_{22}^{\min }}^{N_{22}^{\max }} \mathcal{N}\left(\mathcal{T}_{1}, \mathcal{T}_{2}, N_{22}\right) \mathrm{e}^{-\left(\frac{1}{2} \tilde{\lambda}+\tilde{k}\right) N_{22}},
$$

where the sum is over all values $N_{22}$ which can occur in sandwich geometries with boundary $\mathcal{T}_{1} \cup \mathcal{T}_{2}$. The combinatorial factor $\mathcal{N}\left(\mathcal{T}_{1}, \mathcal{T}_{2}, N_{22}\right)$ counts the number of distinct three-geometries (including the symmetry factor weights) in $[t, t+1]$ for a fixed number of $(2,2)$-tetrahedra.

The Euclideanized amplitude for propagating a spatial geometry $\mathcal{T}_{1}=\mathcal{T}(0)$ at proper time 0 to a later geometry $\mathcal{T}_{2}=\mathcal{T}(t)$ at proper time $t$ is obtained by a $t$-fold iteration of the transfer matrix,

$$
G\left(\mathcal{T}_{1}, \mathcal{T}_{2}, t\right)=\left\langle\mathcal{T}_{2}\left|\hat{T}^{t}\right| \mathcal{T}_{1}\right\rangle
$$

and satisfies the completeness relation

$$
\left\langle\mathcal{T}_{2}\left|\hat{T}^{t}\right| \mathcal{T}_{1}\right\rangle=\sum_{\mathcal{T}}\left\langle\mathcal{T}_{2}\left|\hat{T}^{t_{1}}\right| \mathcal{T}\right\rangle C_{\mathcal{T}}\left\langle\mathcal{T}\left|\hat{T}^{t_{2}}\right| \mathcal{T}_{1}\right\rangle
$$

for any split $t=t_{1}+t_{2}$ of the total time interval.

\section{Integrating out geometries}

Let us first recall the situation in classical 3d gravity, on space-times with compact spatial slices $\Sigma^{(g)}$ of genus $g$. The degrees of freedom of the theory associated with any spatial slice are the geometries (i.e. the spatial metrics $g_{i j}$ modulo spatial diffeomorphisms), the elements of superspace. A priori, they are genuine field degrees of freedom: for example, each metric can be decomposed uniquely (up to a diffeomorphism) into a constant-curvature metric $\bar{g}_{i j}$ and a conformal factor according to $g_{i j}(x)=\mathrm{e}^{2 \lambda(x)} \bar{g}_{i j}(x)$. However, a canonical analysis reveals that the conformal factor $\lambda(x)$ is not a dynamical field degree of freedom, but is completely determined by solving the constraints. What remains is a finite number of so-called Teichmüller parameters (none for $g=0,2$ for $g=1$ and $6 g-6$ for $g>1$ ), coordinatizing the space of constant-curvature metrics for a given compact spatial manifold of genus $g$. This implies that for the spherical case with $\Sigma^{(0)}=S^{2}$ not even a finite number of (classical) degrees of freedom is left after the conformal factor has been fixed

\footnotetext{
${ }^{5}$ We mean here "bulk" degrees of freedom; depending on the choice of boundary conditions on the initial and final spatial slices, surface degrees of freedom may be "liberated".
} 
This raises the question of how these classical properties are reflected in a gravitational path-integral approach of the type we are considering. At the discretized level, we can in principle compute the quantum amplitude between two arbitrary spatial geometries (either at a fixed proper-time distance $t$, or for an arbitrary distance, obtained by summing the discrete propagator (7) over all positive integers $t$ ). In general, this amplitude will not vanish, since there are always many $3 \mathrm{~d}$ quadrangulations interpolating between two given boundaries $\mathcal{T}_{1}$ and $\mathcal{T}_{2}$. It implies that a generic path (i.e. a three-geometry) contributing to the path integral does not obey the classical constraints, which is not particularly surprising. However, what one is really interested in is the behaviour of these amplitudes in the continuum limit.

Previous investigations of the non-perturbative gravitational path integral suggestive of what may be happening in three dimensions. The continuum analysis of [23] found that - subject to a number of plausible conditions - the kinetic term of the conformal factor $\lambda$ is cancelled in the non-perturbative path integral by a FaddeevPopov term in the measure. This means that there is no conformal kinetic term in the effective action, and therefore that $\lambda(x)$ is not a propagating field degree of freedom. This scenario is corroborated by our numerical simulations of $3 \mathrm{~d}$ Lorentzian gravity 18, 19 which did not show any evidence of the conformal divergence closely associated with the kinetic term for the conformal factor.

Motivated by these considerations, we will introduce a vastly reduced set of (Hilbert space) states, by summing over all geometric degrees of freedom of a spatial slice of a given two-volume $N$. Writing $\left|\mathcal{T}_{N}\right\rangle$ for a state corresponding to a quadrangulation with $N$ squares, we define for a fixed spatial topology the state

$$
|N\rangle:=\frac{1}{\sqrt{\sum_{\mathcal{T}_{N}} C_{\mathcal{T}_{N}}}} \sum_{\mathcal{T}_{N}} C_{\mathcal{T}_{N}}\left|\mathcal{T}_{N}\right\rangle .
$$

The normalization factor in front of the sum ensures the orthonormality of the "area states", $\left\langle N \mid N^{\prime}\right\rangle=\delta_{N, N^{\prime}}$. The discrete area $N$ may be thought of as a global conformal degree of freedom. We do not integrate over $N$ in order to keep control over the continuum limit and to be able to compare our results with those of similar continuum approaches (see, for example, 24, 25]). Using the new states (9), we now make the conjecture that

$$
\left\langle N_{14}|\hat{T}| \mathcal{T}_{N_{41}}\right\rangle-\left\langle N_{14}|\hat{T}| \mathcal{T}_{N_{41}}^{\prime}\right\rangle \rightarrow 0 \quad \text { for } \quad N_{14}, N_{41} \rightarrow \infty
$$

Expressed in words, this means that for large areas $N_{41}$ and $N_{14}$ the expectation value $\left\langle N_{14}|\hat{T}| \mathcal{T}_{N_{41}}\right\rangle$ does not depend on which "representative" $\mathcal{T}_{N_{41}}$ is chosen from the set of 2 d quadrangulations with $N_{41}$ squares. The conjecture should be understood in a probabilistic sense: it says that the number of states where (10) is not satisfied should have a slower growth as a function of $N$ than the total number of states $\left|\mathcal{T}_{N}\right\rangle$

\footnotetext{
${ }^{6}$ What is relevant for our current purposes is always a configuration space path integral, and not one in phase space.
} 
(which grows exponentially with $N$ for a given spatial topology). Heuristically one can view (10) as expressing that in the large- $N$ limit the matrix elements $\left\langle\mathcal{T}_{N}|\hat{T}| \mathcal{T}_{N^{\prime}}^{\prime}\right\rangle$ depend not on the two quadrangulations separately, but only on a suitably defined "distance" between $\mathcal{T}_{N}$ and $\mathcal{T}_{N^{\prime}}^{\prime}$, similar to the way the integral $\int \mathrm{d} x f(x-y)$ over the real line is independent of $y$. In principle it is a combinatorial problem to show that (10) is valid, but we have not yet produced such a proof.

An important consequence of property (10) is that the completeness relation (8) continues to hold for the area states $|N\rangle$,

$$
\left\langle N_{2}\left|\hat{T}^{t_{1}+t_{2}}\right| N_{1}\right\rangle=\sum_{N}\left\langle N_{2}\left|\hat{T}^{t_{2}}\right| N\right\rangle\left\langle N\left|\hat{T}^{t_{1}}\right| N_{1}\right\rangle
$$

where we have again assumed that $N_{1}, N_{2}$ and $N$ are all large. We therefore still have a transfer matrix formalism, but with the transfer matrix $\hat{T}$ acting only on the subspace spanned by the linear combinations $\{|N\rangle\}$ of the original Hilbert space. Obviously, the task of diagonalizing the transfer matrix on this reduced space is considerably simplified compared with the original problem.

From now on, we will focus our interest on solving the combinatorics of a single sandwich geometry. We can rewrite relation (6) in an obvious notation as

$$
\left\langle N_{14}|\hat{T}| N_{41}\right\rangle=\mathrm{e}^{-c_{0}-(\tilde{\lambda}-\tilde{k})\left(N_{41}+N_{14}\right)} \sum_{N_{22}=N_{22}^{\min }}^{N_{22}^{\max }} \mathcal{N}\left(N_{41}, N_{14}, N_{22}\right) \mathrm{e}^{-\left(\frac{1}{2} \tilde{\lambda}+\tilde{k}\right) N_{22}}
$$

where

$$
\mathcal{N}\left(N_{41}, N_{14}, N_{22}\right)=\sum_{\mathcal{T}_{N_{41}}, \mathcal{T}_{N_{14}}} \mathcal{N}\left(\mathcal{T}_{N_{41}}, \mathcal{T}_{N_{14}}, N_{22}\right)
$$

denotes the total number of quadrangulations of the space-time between $t$ and $t+1$, including sums over the connectivities of the spatial boundary geometries at $t$ and $t+1$. One may think of $(13)$ as describing the combinatorics of quadrangulating a sandwich geometry with free boundary conditions, except for the areas of the two boundaries which are kept fixed.

In order to simplify the combinatorics further, we introduce boundary cosmological constants $\Lambda_{i}$ and $\Lambda_{f}$ associated with the initial and final boundaries at $t$ and $t+1$. They do not have an immediate physical interpretation and should simply be thought of as convenient book-keeping devices that will be set to zero at the end. They give rise to an additional term

$$
\Delta S=\Lambda_{i} a_{s}^{2} N_{41}+\Lambda_{f} a_{s}^{2} N_{14} \equiv z_{i} N_{41}+z_{f} N_{14}
$$

in the action (3), which will allow us to introduce an asymmetry in the coupling constants multiplying the two areas $N_{41}$ and $N_{14}$. We can use the dimensionless

\footnotetext{
${ }^{7}$ This is analogous to the introduction of external sources into a quantum field-theoretic path integral in order to obtain Green's functions through functional differentiation.
} 
boundary cosmological terms $z_{i}$ and $z_{f}$ to obtain the Laplace transform of the transfer matrix with respect to both the initial and final areas, $N_{i} \equiv N_{41}$ and $N_{f} \equiv N_{14}$, namely,

$$
\left\langle z_{f}|\hat{T}| z_{i}\right\rangle=\sum_{N_{i}, N_{f}} \mathrm{e}^{-z_{i} N_{i}-z_{f} N_{f}}\left\langle N_{f}|\hat{T}| N_{i}\right\rangle
$$

The matrix elements $\left\langle N_{f}|\hat{T}| N_{i}\right\rangle$ are calculated for $z_{i}=z_{f}=0$. From a combinatorial point of view, $\left\langle N_{f}|\hat{T}| N_{i}\right\rangle$ counts the three-geometries $\mathcal{G}$ for given numbers $N_{i}$ and $N_{f}$, each with relative weight $e^{-\left(\frac{1}{2} \tilde{\lambda}+\tilde{k}\right) N_{22}}$, and $\left\langle z_{f}|\hat{T}| z_{i}\right\rangle$ plays the role of a generating function for these numbers. It is usually much easier in combinatorial problems to calculate the generating function rather than the actual numbers. This is well illustrated by both Lorentzian and Euclidean simplicial quantum gravity in dimension two, where boundary cosmological constants are introduced in an analogous manner, and where the associated generating functional greatly simplifies the counting of geometries.

The reason for the simplification is the fact that by going to $\left\langle z_{f}|\hat{T}| z_{i}\right\rangle$ we have achieved totally free boundary conditions, since the constraints of fixed areas $N_{i}$ and $N_{f}$ have been lifted. On the other hand, no information has been lost, since we can in principle always rederive $\left\langle N_{f}|\hat{T}| N_{i}\right\rangle$ by an inverse Laplace transformation from $\left\langle z_{f}|\hat{T}| z_{i}\right\rangle$. Given the one-step propagator $\left\langle N_{f}|\hat{T}| N_{i}\right\rangle$, we can finally obtain the propagator $G\left(N_{i}, N_{f}, t\right)$ for arbitrary times by iterating according to (11).

\section{Extracting the Hamiltonian}

Once the matrix elements $\left\langle N_{f}|\hat{T}| N_{i}\right\rangle$ are known, one may try to extract the continuum Hamiltonian operator $\hat{H}$ of the system by expanding them in the lattice spacing $a$ and then taking $a \rightarrow 0$,

$$
\left\langle N_{f}|\hat{T}| N_{i}\right\rangle=\left\langle N_{f}\left|\mathrm{e}^{-a \hat{H}}\right| N_{i}\right\rangle=\left\langle N_{f}\left|\left(\hat{1}-a \hat{H}+O\left(a^{2}\right)\right)\right| N_{i}\right\rangle
$$

In this way one obtains the quantum Hamiltonian in the " $N$-representation". It is also possible (and usually easier) to extract $\hat{H}$ from the Laplace transform of the onestep propagator, $\left\langle z_{f}|\hat{T}| z_{i}\right\rangle$ (yielding the Hamiltonian in the "dual" z-representation).

Let us illustrate this by a concrete calculation in $2 \mathrm{~d}$ Lorentzian gravity, where the space-time has topology $S^{1} \times[0,1]$. In this case, the generating functional $\left\langle z_{f}|\hat{T}| z_{i}\right\rangle$ is known explicitly [6],

$$
\left\langle z_{f}|\hat{T}| z_{i}\right\rangle=\log \left(\frac{\left(1-\mathrm{e}^{-\left(\lambda_{2}+z_{i}\right)}\right)\left(1-\mathrm{e}^{-\left(\lambda_{2}+z_{f}\right)}\right)}{1-\mathrm{e}^{-\left(\lambda_{2}+z_{i}\right)}-\mathrm{e}^{-\left(\lambda_{2}+z_{f}\right)}}\right) .
$$

\footnotetext{
${ }^{8}$ In line with our construction in dimension three, we consider here the symmetric propagator of [6] with unmarked boundary loops.
} 
The relevant bare couplings in two dimensions are the bulk cosmological constant $\lambda_{2}$ and the boundary cosmological constants $z_{i}$ and $z_{f}$. They are related to the renormalized continuum coupling constants $\Lambda_{2}, \Lambda_{i}, \Lambda_{f}$ by

$$
\lambda_{2}=\log 2+\frac{1}{2} \Lambda_{2} a^{2}, \quad z_{i, f}=\Lambda_{i, f} a .
$$

It is straightforward to expand (17) to lowest non-trivial order in the lattice spacing $a$ and thus obtain the matrix elements $\left\langle\Lambda_{f}|\hat{H}| \Lambda_{i}\right\rangle$. We have

$$
\left\langle\Lambda_{f}|\hat{T}| \Lambda_{i}\right\rangle=\text { analytic }-\log \left(\Lambda_{i}+\Lambda_{f}\right)-a \frac{\Lambda_{2}-\frac{1}{2}\left(\Lambda_{i}^{2}+\Lambda_{f}^{2}\right)}{\Lambda_{i}+\Lambda_{f}}+O\left(a^{2}\right),
$$

where "analytic" refers to a constant term and to terms linear in $\Lambda_{i}$ and $\Lambda_{f}$. By an inverse Laplace transformation we can change variables from the boundary cosmological constants $\Lambda_{i, f}$ to the conjugate (continuum) length variables $L_{i, f}$ of the boundaries. To first order in $a$, one finds

$$
\begin{aligned}
\left\langle L_{f}|\hat{T}| L_{i}\right\rangle & =\int_{-i \infty}^{i \infty} \frac{\mathrm{d} \Lambda_{i}}{2 \pi i} \int_{-i \infty}^{i \infty} \frac{\mathrm{d} \Lambda_{f}}{2 \pi i} \mathrm{e}^{\Lambda_{i} L_{i}+\Lambda_{f} L_{f}}\left\langle\Lambda_{f}|\hat{T}| \Lambda_{i}\right\rangle \\
& =\frac{1}{L_{i}} \delta\left(L_{i}-L_{f}\right)-a\left(-\frac{\mathrm{d}^{2}}{\mathrm{~d} L_{f}^{2}}+\Lambda_{2}\right) \delta\left(L_{i}-L_{f}\right),
\end{aligned}
$$

where we have ignored the analytic terms which lead to non-propagating terms of the form $\delta\left(L_{f}\right) \delta\left(L_{i}\right)$. The appearance of such non-universal terms is familiar from the transfer matrix of $2 \mathrm{~d}$ Euclidean gravity and can be accounted for by a careful treatment of the boundary conditions at $L=0$ [1, 21, 22.

In complete analogy with the definition given in footnote 4 , the symmetry factor of a spatial $S^{1}$-boundary of length $L$ is given by $C_{L}=1 / L$ and there is an orthogonal basis $\{|L\rangle\}$ of the continuum Hilbert space, obeying the normalization conditions

$$
\left\langle L_{1} \mid L_{2}\right\rangle=\frac{1}{L_{1}} \delta\left(L_{1}-L_{2}\right), \quad \int_{0}^{\infty} \mathrm{d} L|L\rangle L\langle L|=\hat{1} .
$$

We deduce that the Hamiltonian operator in the " $L$-representation", acting on functions $\psi(L)=\langle L \mid \psi\rangle$, is

$$
\hat{H}=-\frac{\mathrm{d}^{2}}{\mathrm{~d} L^{2}} L+\Lambda_{2} L
$$

which is hermitian with respect to the measure $L \mathrm{~d} L$, as it should be.

In principle we would like to use the same strategy to determine the quantum Hamiltonian $\hat{H}(A)$ and its spectrum also in the three-dimensional case. Of course we cannot be sure that this will lead to a simple differential operator as a function of the spatial volume, as was the case in $d=2$, (22). Even in two dimensions, there are models inspired by Lorentzian gravity where this is not the case [26]. However, in the $2 \mathrm{~d}$ cases studied so far it has always been possible to turn $H$ into a local differential operator by transforming to a variable different from $L$. 


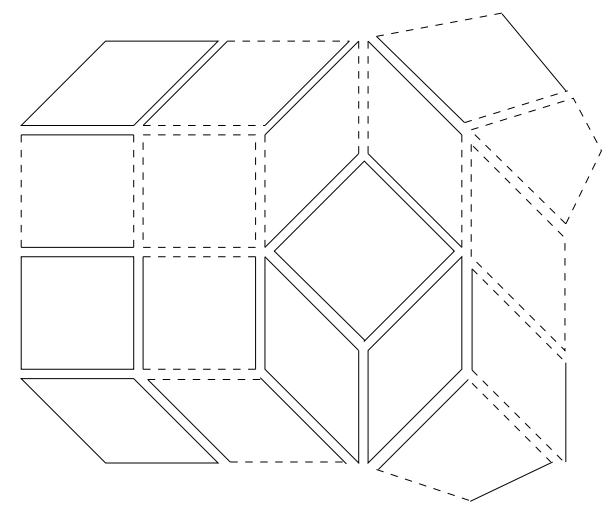

Figure 2: A piece of a typical quadrangulation at $t+1 / 2$. The three types of squares made from solid and dashed lines arise as sections of the (4,1)-, (1,4)- and (2,2)-building blocks.

\section{The matrix model correspondence}

We will now relate the Laplace-transformed one-step propagator (15) to a matrix model. This will be done by showing that the two-dimensional configurations associated with a slicing at half-integer $t$ of a sandwich geometry appear as terms in the perturbative expansion of a hermitian two-matrix model with $A B A B$-interaction. (A similar observation for the original Lorentzian 3d simplicial model was already made in [18].)

Imagine a one-step geometry $\mathcal{G}$ obtained by gluing the three types of building blocks of Fig. 1. The intersection of this three-geometry with the spherical constanttime hypersurface at $t+\frac{1}{2}$ can be visualized as a pattern of squares, whose edges correspond to the intersections of the time-like triangular faces of the $3 \mathrm{~d}$ building blocks with this surface. Let us distinguish the two cases where the time-like triangle has its base either in the $t$ - or the $t+1$-quadrangulation by drawing its intersection at $t+\frac{1}{2}$ as either a solid or a dashed line. Thus a $(4,1)$-pyramid gives rise to a square of solid edges, a (1,4)-pyramid to one of dashed edges and a $(2,2)$-tetrahedron to a square with alternating solid and dashed edges. The way in which these twodimensional building blocks appear in the $S^{2}$-quadrangulation at $t+\frac{1}{2}$ is that they can be glued to each other only along pairs of edges of the same type.

The quadrangulation at $t+\frac{1}{2}$ can thus be viewed as a double-line graph of the kind illustrated in Fig. 2. This type of graph is generated in the large- $M$ limit by the two-matrix model

$$
Z\left(\alpha_{1}, \alpha_{2}, \beta\right)=\int \mathrm{d} A \mathrm{~d} B \mathrm{e}^{-M \operatorname{tr}\left(\frac{1}{2} A^{2}+\frac{1}{2} B^{2}-\frac{\alpha_{1}}{4} A^{4}-\frac{\alpha_{2}}{4} B^{4}-\frac{\beta}{2} A B A B\right)},
$$

where $A$ and $B$ are Hermitian $M \times M$ matrices. By expanding the non-Gaussian part of the exponential in powers of $\alpha_{1}, \alpha_{2}$ and $\beta$ and performing the Gaussian 

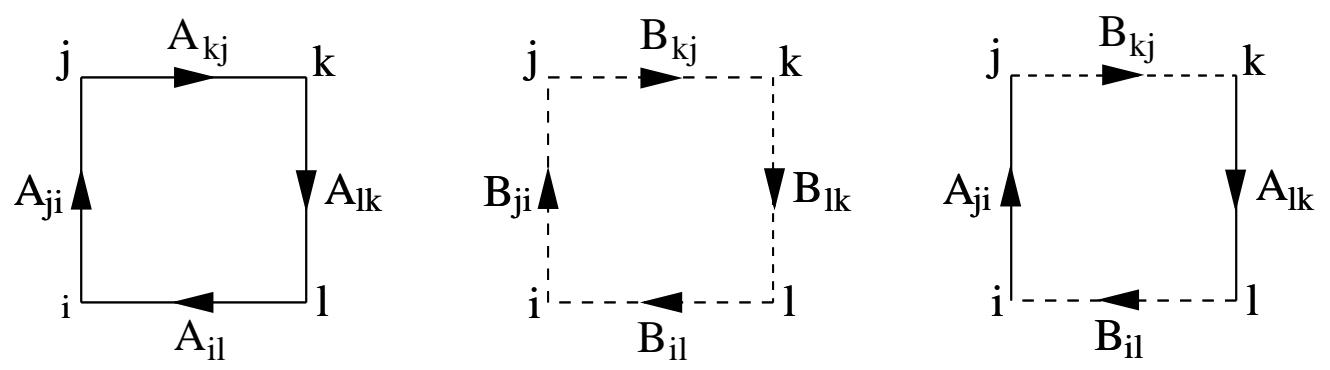

Figure 3: Matrix-model representation of the building blocks at $t+1 / 2$. The gluing rules for the squares are determined by the Gaussian integrations, $\left\langle A_{i j} A_{k l}\right\rangle=\delta_{i l} \delta_{j k}$, $\left\langle B_{i j} B_{k l}\right\rangle=\delta_{i l} \delta_{j k}$, and $\left\langle A_{i j} B_{k l}\right\rangle=0$.

integral we are led by Wick's theorem to a successive gluing of the three kinds of squares described above if we make the index assignments as shown in Fig. 3 .

As usual, the logarithm of the partition function of the model, $M^{2} F\left(\alpha_{1}, \alpha_{2}, \beta\right)=$ $\log Z\left(\alpha_{1}, \alpha_{2}, \beta\right)$, generates only connected quadrangulations, and taking the large- $M$ limit will select those with $S^{2}$-topology. In principle $F$ may be expanded in a power series in $M^{-2}$, with higher-order contributions corresponding to quadrangulations of higher genera. Although we are mainly interested in the spherical limit, anything we say could be repeated for higher-genus surfaces, thus relating to $3 \mathrm{~d}$ gravity on space-times with topology $\Sigma^{(g)} \times[0,1]$. We can now write out the generating function $F$ as an explicit power series,

$$
F\left(\alpha_{1}, \alpha_{2}, \beta\right)=\sum_{N_{41}, N_{14}, N_{22}} \tilde{\mathcal{N}}\left(N_{41}, N_{14}, N_{22}\right) \alpha_{1}^{N_{41}} \alpha_{2}^{N_{14}} \beta^{N_{22}},
$$

where $\tilde{\mathcal{N}}\left(N_{41}, N_{14}, N_{22}\right)$ denotes the number of (connected) spherical quadrangulations described above, including symmetry factors.

Comparing the form of (24) with the previous expressions (12) and (15), and making the identifications

$$
\alpha_{1}=\mathrm{e}^{\tilde{k}-\tilde{\lambda}-z_{i}}, \quad \alpha_{2}=\mathrm{e}^{\tilde{k}-\tilde{\lambda}-z_{f}}, \quad \beta=\mathrm{e}^{-\left(\frac{1}{2} \tilde{\lambda}+\tilde{k}\right)},
$$

one could be tempted to conclude that

$$
F\left(\alpha_{1}, \alpha_{2}, \beta\right) \stackrel{?}{=}\left\langle z_{f}|\hat{T}| z_{i}\right\rangle .
$$

However, this is not correct, since the number of configurations generated by the matrix model is strictly larger than those obtained from the Lorentzian gravity model, that is,

$$
\tilde{\mathcal{N}}\left(N_{41}, N_{14}, N_{22}\right)>\mathcal{N}\left(N_{41}, N_{14}, N_{22}\right)
$$

\footnotetext{
${ }^{9}$ Adopting (25), the limit of vanishing boundary cosmological constants $z_{i, f}$ corresponds to setting $\alpha_{1}=\alpha_{2}$. Unfortunately we cannot put $\alpha_{1}=\alpha_{2}$ before having extracted the matrix elements $\left\langle N_{f}|\hat{T}| N_{i}\right\rangle$ from $F\left(\alpha_{1}, \alpha_{2}, \beta\right)$.
} 

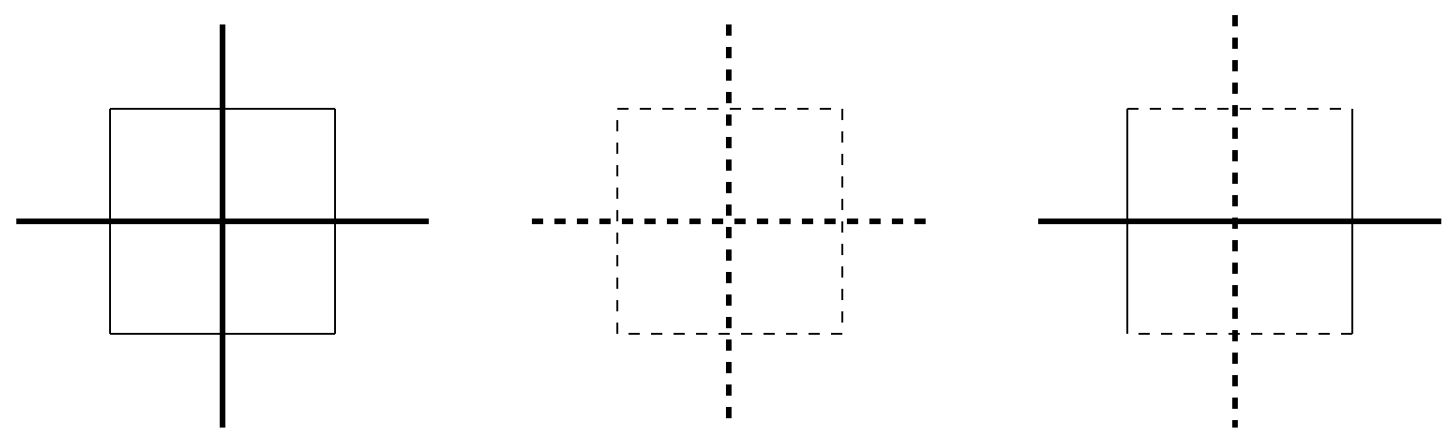

Figure 4: Vertices of $\phi^{4}$-graphs dual to spatial quadrangulations.

This difference is intimately connected to the fact that in the gravity case, the allowed configurations are not so much two-dimensional structures per se as sections of larger, three-dimensional objects whose three-dimensional manifold structure is encoded in the colouring (the dashed and solid lines) of the two-dimensional graph.

The generalization inherent in the matrix model is best described in terms of the graphs dual (in a two-dimensional sense) to the quadrangulations. In this more conventional picture, the terms $\operatorname{tr} A^{4}, \operatorname{tr} B^{4}$ and $\operatorname{tr} A B A B$ are represented by four-valent vertices (placed at the centres of the squares of the original $2 \mathrm{~d}$ quadrangulation) with four outgoing solid lines, four dashed lines or alternating solid-dashed lines (see Fig. (1). The labels $A$ and $B$ are now associated with the dual solid and dashed edges connecting pairs of such vertices.

In order to discuss the regularity conditions that must be satisfied by such a dual graph to qualify as (the dual of) a section of a Lorentzian three-geometry, we define a $A$-loop (a $B$-loop) as a closed sequence of solid (dashed) dual edges with no further solid (dashed) dual links in its interior. This interior region (which has the form of a two-dimensional disc whose boundary is the loop) we call a $A$-domain (a $B$-domain). A dual graph coming from a 3 d Lorentzian geometry then satisfies the following constraints (c.f. App. 2 of [18]):

(1) The two subgraphs formed from only $A$-edges and only $B$-edges must each be connected.

(2) The two separate $A$ - and $B$-subgraphs can have neither tadpoles nor selfenergy subdiagrams. This ensures that they are associated with regular $2 \mathrm{~d}$ simplicial manifolds at the times $t$ and $t+1$ respectively.

(3) The intersection of any pair of $A$ - and $B$-domains cannot be multiply connected. This implies that any pair of vertices, one at time $t$ and one at time $t+1$, of the original quadrangulation cannot be connected by more than one time-like link.

(4) The (one-dimensional) intersection of a $A$-loop with a $B$-domain (and vice 
versa) must be either empty or simply connected. This implies that any three vertices of the original quadrangulation cannot belong to more than one timelike triangle.

Thus we see that the dual graphs coming from the matrix model are considerably more general than those associated with $3 \mathrm{~d}$ simplicial space-times. The generalizations occur in several ways. The matrix-model graphs can have arbitrary numbers of disconnected spherical $A$ - and $B$-subgraphs (the only requirement being that the combined graph is spherical and connected) and each of these may contain (generalized) self-energy diagrams and tadpoles. Furthermore, the $A$ - and $B$-loops can freely meander around each other, with arbitrary numbers of mutual intersections.

We conjecture that the conditions (2)-(4) are not important in the sense that their implementation or otherwise will not affect the continuum properties of the model. We think that they constitute merely a $3 \mathrm{~d}$ generalization of the universal behaviour already observed for $2 \mathrm{~d}$ matrix models. In that case the inclusion of tadpole and self-energy subgraphs leaves the continuum limit unchanged (although it implies an enlargement of the configuration space from genuine $2 \mathrm{~d}$ simplicial manifolds to more general $2 \mathrm{~d}$ combinatorial complexes).

We have checked numerically that the same happens in our $3 \mathrm{~d}$ model. More precisely, we have performed simulations for the original simplicial model with tetrahedral building blocks of [15, 18, 16] where one can formulate conditions completely analogous to (1)-(4) above. Dropping then the constraints (2)-(4), but keeping (1), we found that the key results of [18 remained unaffected. $\square$

The status of condition (1) is different. Recall that in the original representation one obtains the geometry at time $t$ (at time $t+1$ ) from the quadrangulation at $t+1 / 2$ by shrinking all dashed (solid) links to zero. The $A$ - and $B$-graphs of the dual picture at $t+1 / 2$ are of course individually precisely the duals of these twogeometries at integer times. What does it imply for the spatial quadrangulation at $t$ if the dual $A$-graph is disconnected? Using the same "shrinking-prescription", it cannot separate into several pieces, but it does degenerate in the sense of forming a number of connected spherical graphs which touch each other pairwise only in single points. The resulting two-dimensional space is therefore no longer a manifold, but a branched tree of such spherical components.

This phenomenon is illustrated in Fig. : The pictures at the top show two quadrangulations of a two-sphere at time $t+1 / 2$ made of the three types of square-shaped building blocks. As two-dimensional configurations they look rather innocent and regular. However, they are pathological from a three-dimensional point of view.

\footnotetext{
${ }^{10}$ However, one interesting change did occur: in [18] we observed a first-order phase transition for large $\tilde{k}$ (there called $k_{0}$ ) to a phase where successive spatial slices decouple. We viewed the presence of this phase as a discretization artifact irrelevant for the continuum physics. This interpretation is corroborated by our recent simulations without the constraints (2)-(4), where the large- $\tilde{k}$ phase is simply absent. It implies that the generalized model gives in some sense a better representation of continuum physics. This is reminiscent of $2 \mathrm{~d}$ simplicial quantum gravity, where the continuum limit is approached much faster if self-energy and tadpole graphs are allowed.
} 

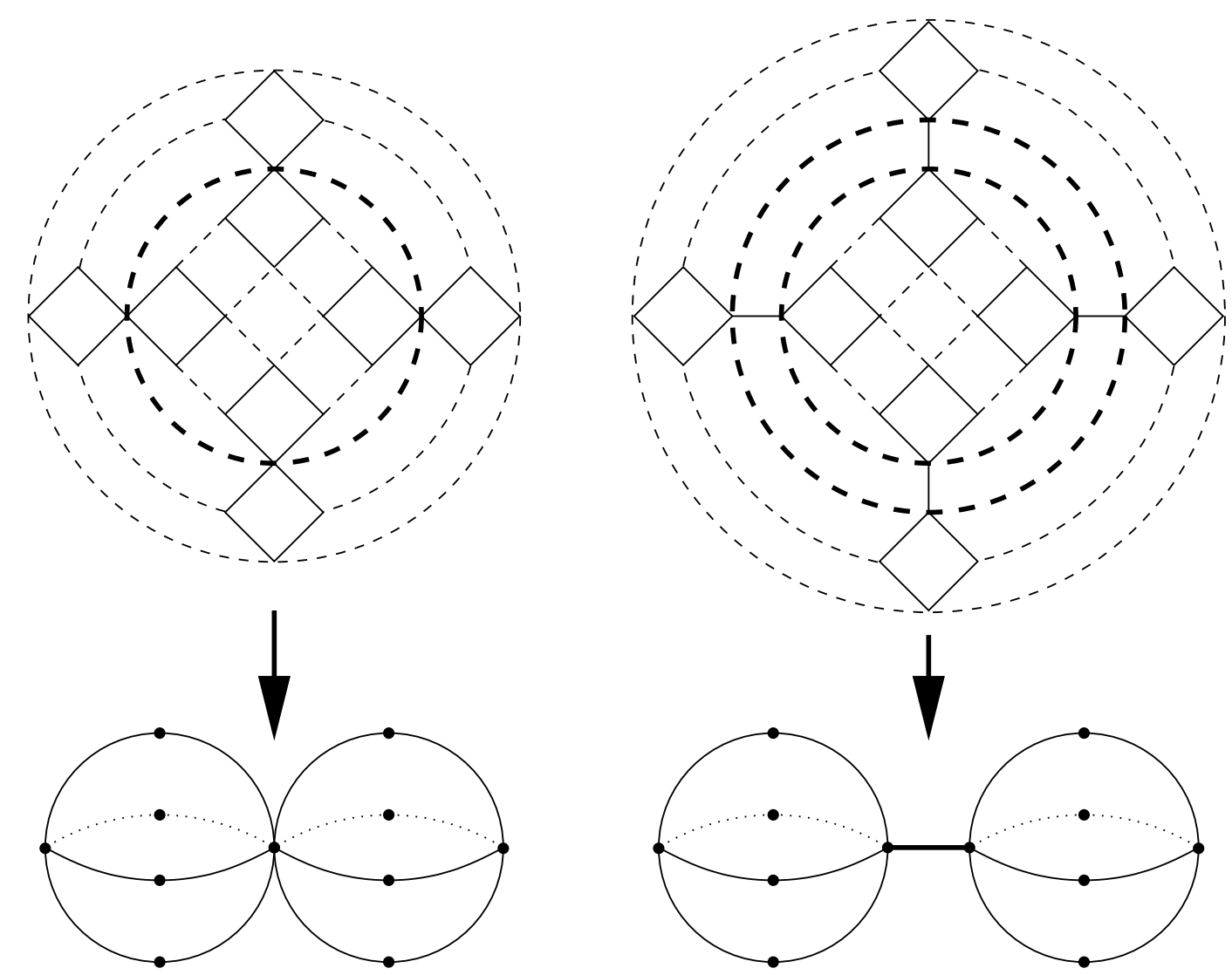

(a)

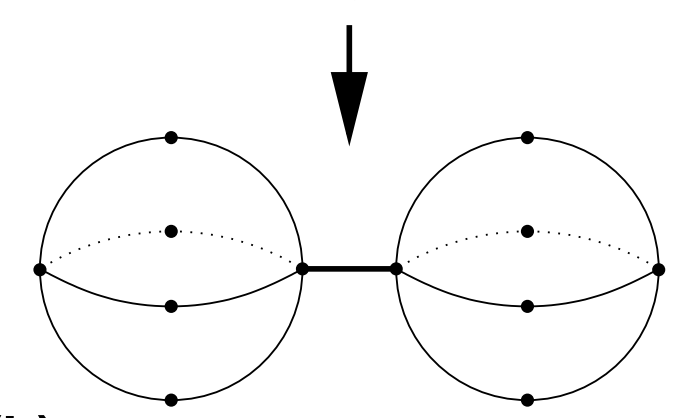

(b)

Figure 5: Examples of matrix-model configurations at $t+1 / 2$ which are not allowed in the original Lorentzian gravity model and which result in geometries with wormholes at time $t$. Shrinking the dashed links to zero, one obtains the two-geometries at the bottom. The thick dashed lines in the quadrangulations at the top are contracted to touching points or to points along one-dimensional wormholes.

Drawing the dual graphs, one finds in both cases that the $A$-subgraph, made of solid dual edges, consists of two components. Each component is dual to a quadrangulation of a two-sphere by four (solid-line) squares. When shrinking away the dashed links of the original quadrangulation, the "necks" (indicated by the thick dashed circular lines) between the two spheres are gradually pinched to points. If there is just one neck, the two spheres will touch in a point (Fig. 5a). If there are several concentric necks, with annuli of dashed-solid squares (corresponding to closed rings of (2,2)-tetrahedra) in between, this process generates one-dimensional "wormholes", as illustrated in Fig. 5b.

Similar so-called "touching"-interactions have been studied in the context of twodimensional matrix models [27, 28], and are closely related to these wormholes (see Sec. 6 for a detailed discussion). Although configurations of this type are already 
present in ordinary matrix models of Euclidean $2 \mathrm{~d}$ gravity, the explicit introduction of such an interaction term in the action (and an associated coupling constant) allows us to increase their weight and thereby control their abundance. Depending on the value of the coupling constant, one can obtain either the ordinary universal behaviour of two-dimensional Euclidean gravity, or a modified critical behaviour, described in more detail below. We will see that in our 3d-gravitational reinterpretation of the matrix model the presence of wormholes and touching-interactions is governed by the value of the bare gravitational coupling constant $\tilde{k}$.

Let us finally note that the above discussion about degenerate geometries could be repeated verbatim for the cubic matrix model with partition function

$$
Z\left(\alpha_{1}, \alpha_{2}, \beta\right)=\int \mathrm{d} A \mathrm{~d} B \mathrm{e}^{-M \operatorname{tr}\left(\frac{1}{2} A^{2}+\frac{1}{2} B^{2}-\frac{\alpha_{1}}{3} A^{3}-\frac{\alpha_{2}}{3} B^{3}-\frac{\beta}{2} A B A B\right)},
$$

which is associated with the original $3 \mathrm{~d}$ simplicial gravity model described in [15, 16] (with (3,1)- and (1,3)-tetrahedra instead of (4,1)- and (1,4)-pyramids). The only reason why we prefer to use the quartic model defined by (23) is that it has been solved for $\alpha_{1}=\alpha_{2}$ [20]. We would expect from universality arguments that the models given by (23) and (28) lead to the same continuum physics.

\section{Reinterpreting the matrix model}

We have seen in the previous section that 3d Lorentzian "sandwich" geometries can be put into correspondence with a subclass of $2 \mathrm{~d}$ graphs generated by a quartic matrix model with $A B A B$-interaction. We have also argued that this generalization is potentially relevant, in the sense of the two models having a different phase structure.

The phase structure of the three-dimensional Lorentzian model has been investigated numerically in [18, 19]. For given gravitational coupling $\tilde{k}$, there is a critical value $\tilde{\lambda}_{c}(\tilde{k})$ which is to be approached from above, that is, from the region $\tilde{\lambda}>\tilde{\lambda}_{c}$ of the cosmological constant where the partition function converges. In the original simulations we also observed a first-order transition for large $\tilde{k}$ when moving along the critical line $\tilde{\lambda}_{c}(\tilde{k})$. As already mentioned in footnote 10, this transition is a discretization artifact which disappears when one relaxes some of the manifold constraints. What emerges as the phase structure for 3d Lorentzian gravity is therefore simply that of a single phase (at least in the range of coupling constants accessible to our computer simulations) where the taking of the continuum limit coincides with the tuning of the cosmological constant to its critical value, much as in two-dimensional models of quantum gravity. The role of the gravitational coupling $\tilde{k}$ is merely to set an overall scale for the system, without affecting its continuum properties, as we have argued in [18].

What we want to explore presently is the physical interpretation in terms of three-geometry of the generalized model (where multiply-connected $A$ - and $B$-graphs 


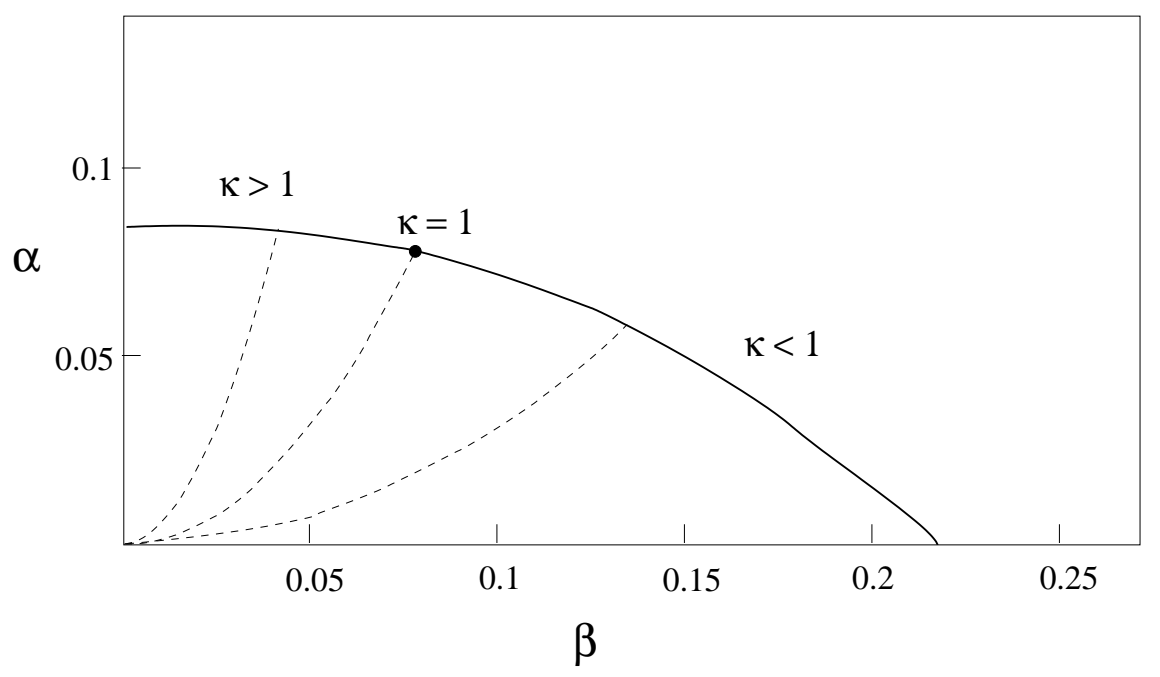

Figure 6: The phase diagram of the $A B A B$-model, according to [20], with the critical line and the critical point at $\alpha=\beta=\frac{1}{4 \pi}$ (corresponding to $\kappa=1$ ). One way of approaching the critical line is through fine-tuning of the cosmological constant $\tilde{\lambda}$ along lines of constant $\tilde{k}$, shown as dashed curves. To end up at the critical point, one should move along the line of constant $\tilde{k}_{c}=-\frac{1}{3} \ln \beta_{c} \simeq 0.85$.

are allowed to occur as the spatial sections at half-integer times) and of its phase structure. This is of course the two-matrix model defined by eq. (23). Let us consider first the case of vanishing boundary cosmological constants, $z_{i, f}=0$. Although we will not be able to construct the Hamiltonian or the propagator (c.f. footnote 9), we can nevertheless discuss the phase diagram of the model. We will comment later on the general matrix model with $z_{i}, z_{f} \neq 0$.

Physically this choice of couplings implies that we are studying the geometric fluctuations between two successive spatial slices with free boundary conditions. As was clear from the computer simulations in [18], the behaviour of this one-step system determines the phase structure of the discretized theory. Other than that, the choice $z_{i, f}=0$ has the great advantage that the corresponding matrix model has already been solved. Details of the solution can be found in the paper of V. Kazakov and P. Zinn-Justin [20]. Their analysis of the matrix model with $\alpha_{1}=\alpha_{2}=\alpha$ can be summarized as follows. Let us fix a ratio $\kappa=\alpha / \beta$ and gradually increase the values of $\alpha$ and $\beta$ away from zero. The large- $M$ limit of the matrix model is defined for sufficiently small $\alpha$ and $\beta$, and the model becomes critical at a point $\left(\beta_{c}(\kappa)\right.$, $\left.\alpha_{c}(\kappa)=\kappa \beta_{c}(\kappa)\right)$, giving rise to a critical line in the $(\beta, \alpha)$-plane (see Fig. 6). Along it one finds two phases, separated by a second-order phase transition at $\kappa=1$, with $\alpha_{c}(1)=\beta_{c}(1)$. In the context of two-dimensional gravity, they were given the following interpretation [20]: the phase of $\kappa>1$ with $\alpha_{c}(\kappa)>\beta_{c}(\kappa)$ can be viewed as ordinary two-dimensional Euclidean gravity, with central charge $c=0$. In this phase the relation between the length $l$ of a "typical" loop of $A$-links (or $B$-links) and the 
area $\mathcal{A}$ of the two-dimensional surface enclosed by the loop is $l^{2} \sim \mathcal{A}$. By contrast, in the other phase (which also has $c=0$ ), the scaling is anomalous, $l^{4 / 3} \sim \mathcal{A}$.

One can understand these two situations by simply looking at the two limits $\beta=0$ and $\alpha=0$. By setting $\beta=0$ we switch off the $A B A B$-interaction, leading to a decoupled system of two $\phi^{4}$ one-matrix models, whose critical behaviour is individually that of an ordinary $2 \mathrm{~d}$ Euclidean gravity system. Setting $\alpha=0$, we obtain a matrix model whose only interaction comes from the term $\operatorname{tr} A B A B$. This model was first solved in 29 by mapping it to the so-called dense loop phase of the $O(1)$-matrix model on random graphs."1

\subsection{Intermezzo: touching-interactions}

We will now reinterpret the results described above in the context of three-dimensional Lorentzian gravity. Recall our earlier discussion in Sec. 5, where we showed that from a three-dimensional point of view a generic feature of the matrix-model configurations is the presence of touching points and wormholes in the associated spatial slices. The matrix model therefore describes transitions between two-geometries with a tree-like structure, consisting of regular quadrangulations of two-spheres, pairwise connected by one-dimensional wormholes of length $l \geq 0$ (Fig. 5).

Let us for a moment revert back to a pure matrix-model language. For the sake of definiteness, we will concentrate on a quadrangulation at time $t$ made from solid squares. We will now show that the effective weight associated with the mutual touching points of the two-spheres making up the tree-configuration is obtained by integrating out the $B$-matrices. While this cannot be done explicitly as long as the $\operatorname{tr} B^{4}$ term is present, it is instructive to do the integration for the general matrix model (23) with $\alpha_{2}=0 . \square$ In this case, the action is Gaussian in the $B$-matrix and we obtain

$$
\begin{aligned}
Z\left(\alpha_{1}, \alpha_{2}=0, \beta\right) & =\int \mathrm{d} A \mathrm{~d} B \mathrm{e}^{M\left(-\frac{1}{2} \operatorname{tr}\left(A^{2}+B^{2}\right)+\frac{\alpha_{1}}{4} \operatorname{tr} A^{4}+\frac{1}{2} \beta \operatorname{tr} A B A B\right)} \\
& =\int \mathrm{d} A\left(\operatorname{det}\left(I-\beta A^{\mathrm{T}} \otimes A\right)\right)^{-\frac{1}{2}} \mathrm{e}^{M\left(-\frac{1}{2} \operatorname{tr} A^{2}+\frac{\alpha_{1}}{4} \operatorname{tr} A^{4}\right)} \\
& =\int \mathrm{d} A \mathrm{e}^{M\left(-\frac{1}{2} \operatorname{tr} A^{2}+\frac{\alpha_{1}}{4} \operatorname{tr} A^{4}+\frac{1}{2 M} \sum_{k=1}^{\infty} \frac{\beta^{k}}{k} \operatorname{tr} A^{k} \operatorname{tr} A^{k}\right)},
\end{aligned}
$$

which now describes a particular one-matrix model. Terms like $\operatorname{tr} A^{k} \operatorname{tr} A^{k}$ are usually referred to as "touching-interactions". How can their effect be visualized in

11 The $O(1)$-model on random graphs is known to correspond to $c=0$ quantum gravity, but with two distinct phases [30, 31, 32], not unlike those of the $A B A B$-model. In a continuum interpretation, these result from assigning different boundary operators to the theory. The dilute loop phase corresponds to ordinary $2 \mathrm{~d}$ Euclidean quantum gravity where boundary lengths scale according to $l^{2} \sim \mathcal{A}$, while in the dense phase one has $l^{4 / 3} \sim \mathcal{A}[31,32]$.

${ }^{12}$ For $\alpha_{2} \neq 0$ there will be other touching interactions beyond the ones described below. 


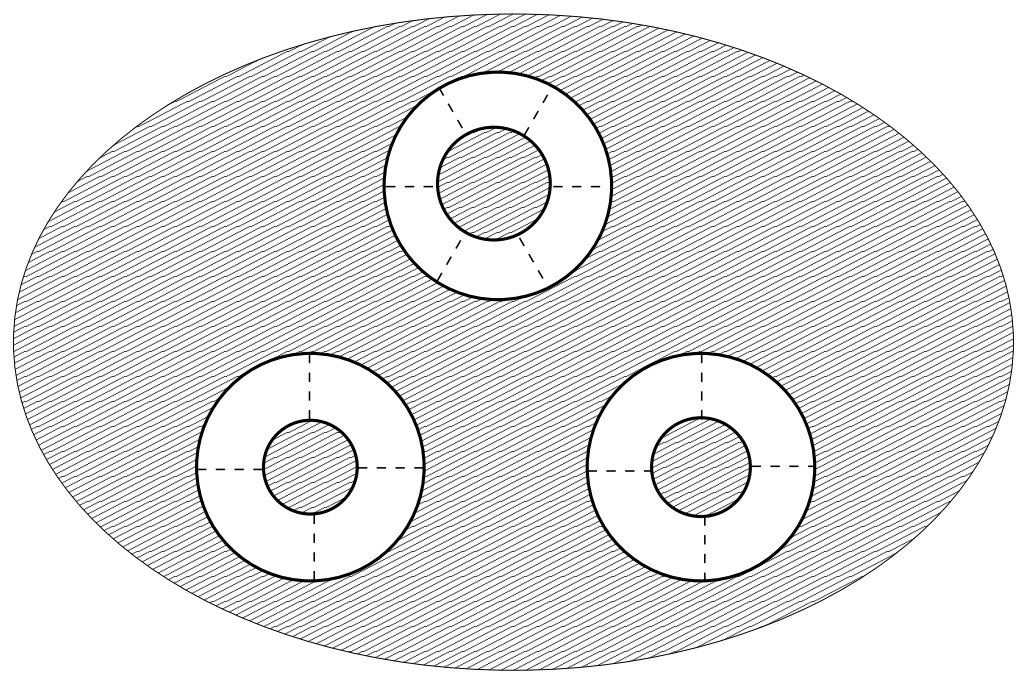

Figure 7: A typical configuration generated by the matrix model (23) for $\alpha_{2}=0$. By integrating out the $B$-matrices one obtains touching terms $\operatorname{tr} A^{k} \operatorname{tr} A^{k}$ (here, $k=4$ and 6 ). Their effect is to identify the boundary links of the solid-square components pairwise across the "gaps" formed by the solid-dashed squares, as described in the text.

geometric terms? Firstly, in the same way as $\operatorname{tr} A^{4}$ represents a square of solid edges, a term $\operatorname{tr} A^{k}$ in the action can be thought of as a $k$-gon. Expanding the action and performing Wick-contractions corresponds to gluing such polygons together by identifying their links pairwise. Imagine that during this process we had constructed a surface glued from various polygons, with a boundary consisting of $k$ links. By performing the appropriate Wick-contractions, we could now glue a $k$-gon (represented by $\operatorname{tr} A^{k}$ ) to this boundary and close off the surface.

By extension, we can determine the effect of a term like $\operatorname{tr} A^{k} \operatorname{tr} A^{k}$. Imagine a pair of surfaces made from polygons, both with a boundary of length $k$. By contracting with $\operatorname{tr} A^{k} \operatorname{tr} A^{k}$, we can close off the two surfaces. Because this happens simultaneously for both of the surfaces, we associate by this process a $k$-gon of the first surface with another $k$-gon of the second surface, which we may think of as a touching point between the two geometries (for example, located at the centres of the $k$-gons).

This is illustrated by Fig. [7, which shows a typical quadrangulation at $t+1 / 2$ appearing in the matrix model with $\alpha_{2}=0$ (so no dashed squares are present), before performing the $B$-integration. The shaded areas represent components made of solid squares, where the outer boundary should be thought of as a single point, since the entire geometry is spherical. Integrating out the $B$-matrices has precisely the effect of generating terms $\operatorname{tr} A^{k} \operatorname{tr} A^{k}$ and identifying the solid boundary links pairwise (each link with its partner lying opposite in a solid-dashed-solid-dashed square). Within the one-matrix model it is of course a matter of convention how 
one wants to think of the pairwise associations introduced by the terms $\operatorname{tr} A^{k} \operatorname{tr} A^{k}$, whether as microscopic touching points between the two closed-off surfaces or simply as regular gluings of one boundary of length $k$ to another. However, it is natural to think of them as creating a connection between the surfaces. There is a factor $1 / M$ associated with each touching interaction, and it is well-known that to leading order in $M$ one obtains through "touching and connecting" only two-geometries with an overall spherical topology, and no spaces of higher genus. Collapsing all the genuinely two-dimensional parts of this two-geometry to one-dimensional lines, we would obtain a branched polymer, i.e. a one-dimensional tree structure without any closed loops.

What is our motivation for this excursion into touching-interactions? The general $A B A B$-model does not in itself suggest an immediate physical interpretation in terms of spatial or space-time geometry. However, with the benefit of hindsight, one could have taken the presence of the limiting case $\beta=0$ (where one has two independent copies of Euclidean $2 \mathrm{~d}$ gravity) as an indication that switching on the $\beta$ coupling will lead to a theory of two interacting two-geometries. The situation where such an interaction occurs naturally is of course when the two-geometries appear as neighbouring embedded spatial slices within a 3d space-time, with their interaction dictated by the 3d Einstein action. Given the three-dimensional interpretation, it is then natural to determine the effect of the neighbouring two-geometry by integrating out the corresponding degrees of freedom in the partition function (for instance, the $B$-matrices). As we have seen, this effectively introduces touching-interactions in a $2 \mathrm{~d}$ slice which are dictated by $\beta$, where $\beta$ in turn depends on the coupling constants $\tilde{\lambda}$ and $\tilde{k}$ of three-dimensional quantum gravity.

Another related point that follows from the three-dimensional interpretation is that it gives us a precise geometric way of obtaining a two-geometry at time $t$ (or $t+1$ ) from a general matrix-model configuration at time $t+1 / 2$ by the shrinkingprescription already mentioned in Sec. 5. This may be visualized as a continuous process where the lengths of either the dashed or the solid links are gradually shrunk to zero. Although this prescription was originally invented to describe the wellbehaved geometries of $3 \mathrm{~d}$ Lorentzian quantum gravity, it works just as well for the more general configurations generated by the matrix model. We have already seen explicit examples in Sec. 2, where this led to the creation of spatial wormholes (Fig. 5).

What is important to notice is that from a three-dimensional point of view, such wormholes and the touching-interactions described in this subsection are really two sides of the same coin. That is, taking a general configuration of the $A B A B-$ model which is not allowed in the original Lorentzian model, one will find both types of degeneracy when applying the shrinking-prescription. We illustrate this with a simple matrix-model graph in Fig. 8. We start at time $t+1 / 2$ from an extended version of Fig. 5b, where the shaded areas indicate regular dashed quadrangulations. Since these will all vanish when we shrink away the dashed edges, we obtain the same wormhole geometry at time $t$ as before. On the other hand, if we now shrink 

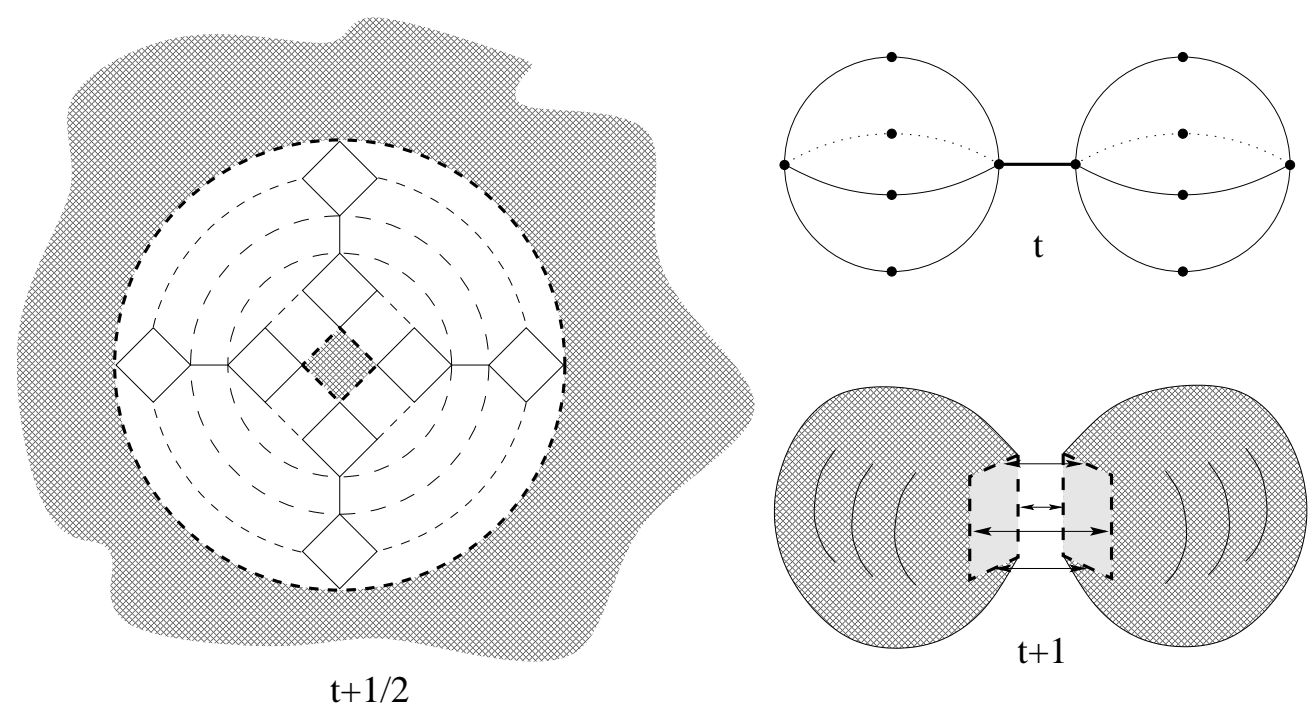

Figure 8: A configuration of the $A B A B$-matrix model, giving rise to a two-geometry with a spatial wormhole at time $t$ and a two-geometry with "touching-interaction" at time $t+1$. Viewed as part of a Lorentzian space-time, this configuration is degenerate.

the solid edges to zero, we are left with two pieces of dashed quadrangulations, each with a boundary of dashed links of length four (the thick dashed lines in Fig. 8). We then must identify the boundary links pairwise, as indicated by the arrows. This is clearly the same mechanism that was at play in the touching-interactions, now generalized to a situation with $\alpha_{2} \neq 0$. Although the resulting configuration at time $t+1$ is (unlike the wormhole at $t$ ) perfectly well-behaved as a two-geometry, it is not acceptable from our original three-dimensional point of view because of the way it is "embedded" in the three-dimensional space-time. We will in the following not distinguish between those two types of geometric degeneracy and simply refer to them as "wormholes" [T

\subsection{Phase structure in the presence of touching-interactions}

Having established the relation with one-matrix models containing (generalized) touching-interactions, let us try to understand what we can learn from the known phase structure of these models. There are three phases when touching-interactions are added to the usual $\operatorname{tr} A^{4}$-interaction. In phase I the touching-interaction plays no role, and the critical behaviour is determined by the $\operatorname{tr} A^{4}$-interaction. A typical configuration (with probability one in the scaling limit) is a connected, spherical quadrangulation of the type that dominates Euclidean $2 \mathrm{~d}$ gravity.

\footnotetext{
${ }^{13}$ This fits with the terminology used in matrix models, where the touching points brought about by the touching-interactions are also sometimes thought of as "wormholes".
} 
In phase II, the touching- and the $\operatorname{tr} A^{4}$-interactions coexist and compete. $\mathrm{A}$ typical two-geometry consists of many spherical $2 \mathrm{~d}$ "baby"-universes of all sizes, which are connected to each other at touching points, thus forming a "blown-up" branched polymer"4. As was first realized in [34, the critical behaviour results from an interplay between the properties Euclidean gravity and those of branched polymers (see also [35).

Quite surprisingly, all critical aspects of this phase can be understood from continuum quantum Liouville theory (which is usually thought to describe only phase I) [36, 37, 38]. Let us review this briefly. Consider a conformal field theory in dimension two with a fixed background metric $\hat{g}$, and let $\Phi$ be a spinless primary field with scaling dimension $\Delta_{\Phi}^{(0)}$. Then the "one-point" function for $\Phi$ scales as

$$
F_{\Phi}^{(0)}(\mathcal{A})=\left\langle\int \mathrm{d}^{2} \xi \sqrt{\hat{g}(\xi)} \Phi(\xi)\right\rangle_{C F T} \sim \mathcal{A}^{1-\Delta_{\Phi}^{(0)}}
$$

where $\mathcal{A}=\int_{\Sigma} \mathrm{d}^{2} \xi \sqrt{\hat{g}(\xi)}$ is the area of the underlying manifold $\Sigma$, and the expectation value is taken in the conformal field theory. Coupling this theory to $2 \mathrm{~d}$ Euclidean quantum gravity, the metric $g$ will be allowed to fluctuate. We can decompose a general metric $g$ into $g=e^{\phi} \hat{g}$, where $\phi(\xi)$ is the conformal field. In conformal gauge, the integration over the fluctuating metric becomes an integration over the conformal factor $\phi$, weighted by the Liouville action $S_{L}[\phi]$, while the spatial integral appearing in the one-point function is changed according to

$$
\int \mathrm{d}^{2} \xi \sqrt{\hat{g}(\xi)} \Phi(\xi) \longrightarrow \int \mathrm{d}^{2} \xi \sqrt{\hat{g}(\xi)} \mathrm{e}^{\beta_{\Phi} \phi(\xi)} \Phi(\xi)
$$

The dressing exponent $\beta_{\Phi}$ is determined by requiring that the "dressed" operator $e^{\beta_{\Phi} \phi} \Phi$ have conformal dimension dimension $(1,1)$, so that it can be integrated over the two-dimensional surface without breaking conformal invariance. This leads to the relation

$$
\beta_{\Phi}\left(\beta_{\Phi}+Q\right)=-2+2 \Delta_{\Phi}^{(0)}, \quad Q=\sqrt{\frac{25-c}{3}} .
$$

The special case of the unit operator $\Phi=1$ corresponds to $\Delta_{1}^{(0)}=0$, with dressing exponent $\beta_{\mathbf{1}}$. The one-point function for the gravity-coupled theory is defined by

$$
\begin{aligned}
F_{\Phi}(\mathcal{A}) & =\left\langle\int \mathrm{d}^{2} \xi \sqrt{\hat{g}(\xi)} \mathrm{e}^{\beta_{\Phi} \phi(\xi)} \Phi(\xi)\right\rangle_{Q G} \\
& :=\frac{\int \mathcal{D} \phi \mathrm{e}^{\frac{c-25}{48 \pi^{2}} S_{L}[\phi]} \delta\left(\int \mathrm{d}^{2} \xi \sqrt{\hat{g}} \mathrm{e}^{\beta_{\mathbf{1}} \phi}-\mathcal{A}\right)\left\langle\int \mathrm{d}^{2} \xi \sqrt{\hat{g}} \mathrm{e}^{\beta_{\Phi} \phi} \Phi\right\rangle_{C F T}}{Z(\mathcal{A})},
\end{aligned}
$$

where $Z(\mathcal{A})$ is the partition function with fixed area $\mathcal{A}$ (enforced by including the same $\delta$-function that appears in the numerator of (33)). By requiring that $F_{\Phi}(\mathcal{A})$ be

\footnotetext{
${ }^{14}$ Further information about the properties of branched polymers can be found in 33 .
} 
independent of the fiducial background metric $\hat{g}$, a change of integration variables $\phi \rightarrow \phi+\frac{1}{\beta_{1}} \log \mathcal{A}$ leads to

$$
F_{\Phi}(\mathcal{A})=\mathcal{A}^{\beta_{\Phi} / \beta_{1}} F_{\Phi}(1)
$$

In analogy with (30), we now define the critical exponent $\Delta_{\Phi}$ of $\Phi$ for the gravitycoupled theory by

$$
F_{\Phi}(\mathcal{A}) \sim \mathcal{A}^{1-\Delta_{\Phi}}
$$

Combining this with relation (34), one derives

$$
\Delta_{\Phi}=1-\frac{\beta_{\Phi}}{\beta_{1}}
$$

For our present purposes it is important to note that eq. (32) has two solutions, namely,

$$
\beta_{\Phi}^{( \pm)}=\frac{-(25-c) \pm \sqrt{(25-c)\left(1-c+24 \Delta_{\Phi}^{(0)}\right)}}{6 Q} .
$$

In conventional quantum Liouville theory one works with $\beta_{\Phi}^{(+)}$because this choice ensures that in the "classical" limit $c=-\infty$ (classical in the sense that the fluctuations of the Liouville field in (33) are completely surpressed) the scaling dimensions of the model without gravity coupling are recovered,

$$
\Delta_{\Phi} \rightarrow \Delta_{\Phi}^{(0)} \text { for } c \rightarrow-\infty
$$

as can easily be verified from formulas (37) and (36).

It is a remarkable fact that all critical exponents which can be calculated in phase II of the matrix model with touching-interactions are obtained by replacing $\beta_{\Phi}^{(+)} \rightarrow \beta_{\Phi}^{(-)}$, changing the scaling relations according to

$$
F_{\Phi}^{(+)}(\mathcal{A}) \sim \mathcal{A}^{\beta_{\Phi}^{(+)} / \beta_{1}^{(+)}} \longrightarrow F_{\Phi}^{(-)}(\mathcal{A}) \sim \mathcal{A}^{\beta_{\Phi}^{(-)} / \beta_{1}^{(+)}}
$$

The implications of this prescription can be illustrated by considering the simplest boundary operator,

$$
\ell=\oint_{\partial \Sigma} \mathrm{d} s \sqrt{\hat{e}(s)}
$$

which measures the length of the boundary of the two-dimensional manifold $\Sigma$ as a function of the induced metric $\hat{e}(s)$ on $\partial \Sigma$. Unlike in matrix models, it is somewhat awkward to introduce boundary operators like (40) in Liouville theory. Nevertheless, $\ell$ can be treated along similar lines as the operator $\Phi$ in (31) above. The expression one obtains for the gravitationally dressed version of the length operator is

$$
\ell=\oint \mathrm{d} s \sqrt{\hat{e}(s)} \mathrm{e}^{\beta_{\ell}^{(+)} \phi(s)}, \quad \beta_{\ell}^{(+)}=\frac{1}{2} \beta_{\mathbf{1}}^{(+)} .
$$


From the point of view of conformal field theory it is rather surprising that the "naive" value $\beta_{\ell}=\frac{1}{2} \beta_{1}$ (i.e. the boundary scales like the square root of the bulk) is correct. However, as can be read off from (34), this does lead to the canonical scaling

$$
\left\langle\ell^{(+)}(\mathcal{A})\right\rangle_{Q G} \sim \mathcal{A}^{\beta_{\ell}^{(+)} / \beta_{1}}=\mathcal{A}^{1 / 2}
$$

already known from matrix-model considerations.

To determine the scaling relevant in phase II, we should now replace the operator $\beta_{\ell}^{(+)}$. It turns out that the correct substitution for this particular boundary operator is given by

$$
\beta_{\ell}^{(+)} \longrightarrow \beta_{\ell}^{(-)}=\frac{1}{2} \beta_{1}^{(-)} .
$$

This leads to the scaling behaviour

$$
\left\langle\ell^{(-)}(\mathcal{A})\right\rangle_{Q G} \sim \mathcal{A}^{\beta_{\ell}^{(-)} / \beta_{\mathbf{1}}^{(+)}}=\mathcal{A}^{3 / 4}
$$

implying that the boundary scales anomalously in terms of the area. This anomalous scaling is clearly a reflection of the additional fractal structure introduced by the touching-interactions. Note that it coincides with the spectral dimension of branched polymers [39, 40.

The scaling (44) coincides with the scaling behaviour observed in the $A B A B$ matrix model in the phase with $\kappa<1$, and mentioned already earlier in Sec. 6. This corroborates our interpretation of this model in terms of interacting two-geometries. Furthermore, it agrees with the relation between loop length and area found in the dense phase of the $O(1)$-model, where the replacement (43) was first noticed [32].

Lastly, the critical behaviour in phase III of the matrix model with touchinginteractions is characterized by a complete dominance of branched polymers. The size of the individual spherical components making up the $2 \mathrm{~d}$ universe never exceeds the cutoff scale. This phase does not seem to have an analogue in the $A B A B$-matrix model.

\subsection{Summary of Sec. 6}

Let us summarize what we have learned about the three-dimensional geometric interpretation of the $A B A B$-model. To this end, we will first translate its phase diagram (for $\alpha_{1}=\alpha_{2}$ ) to the $\tilde{k}-\tilde{\lambda}$-plane of the gravitational couplings. We deduce from (25) that

$$
\frac{\alpha}{\beta^{2}}=\mathrm{e}^{3 \tilde{k}} \quad \text { and } \quad \frac{1}{G_{N}} \sim \tilde{k}=\frac{1}{3} \log \frac{\kappa}{\beta_{c}(\kappa)},
$$

where the second relation holds along the critical line (the points where a nontrivial continuum limit exists). The shaded areas in the two phase diagrams of Fig. 9 indicate the region in which the partition function converges. The dashed curves in both diagrams show an approach to the critical line along a curve of constant $\tilde{k}$. Moving from left to right along the critical curve of the left phase diagram (so that 

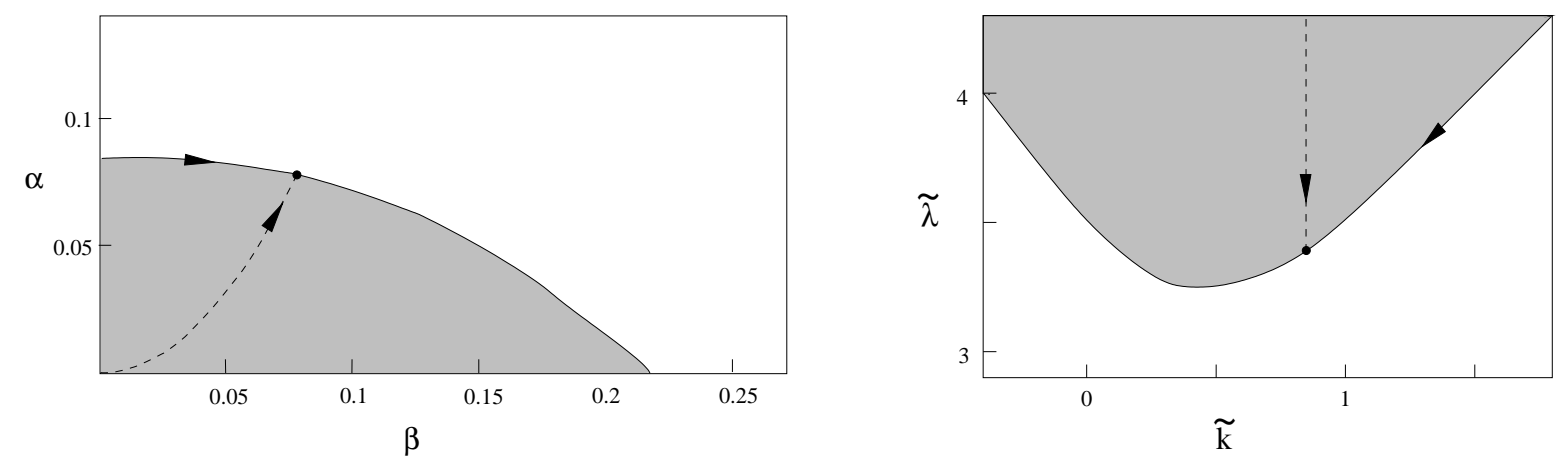

Figure 9: Translating the phase diagram of the $A B A B$-matrix model in terms of the original coupling constants $\alpha$ and $\beta$ (left) to the couplings $\tilde{k}$ and $\tilde{\lambda}$ associated with its interpretation in terms of $3 \mathrm{~d}$ Lorentzian gravity (right).

$\beta$ increases and $\kappa$ decreases) translates into a motion from right to left along the critical line of the "gravitational" phase diagram (that is, towards a smaller $\tilde{k}$ or a larger bare Newton's constant $G_{N}$ ).

In terms of space-time geometry, at the point $G_{N}=0$ (corresponding to $\beta=0$ ) subsequent spatial slices are completely decoupled. No information can be propagated in time and the system has no interesting three-dimensional properties. Increasing $G_{N}$ away from zero, we enter the phase $\kappa>1$ of the matrix model. Interactions between neighbouring spatial slices of the $3 \mathrm{~d}$ universe become possible 5 , and the geometrical properties of a typical spatial slice at integer- $t$ resemble those generated in $2 \mathrm{~d}$ Euclidean quantum gravity. It is very suggestive to identify this phase with that of Lorentzian gravity defined in [15, 16], since the simulations of [18, 19] revealed both the existence of extended three-dimensional space-times (indicative of correlations in time-direction), and evidence that the Hausdorff dimension of the spatial slices is $d_{H}=4$, in agreement with that of 2 d Euclidean gravity.

Apparently, the phase with $\kappa \leq 1$ cannot be realized in the Lorentzian manifold model because it does not allow for the creation of "wormholes" " B. By contrast, no such restriction exists in the $A B A B$-matrix model, and by gradually increasing Newton's constant $G_{N}$ such wormhole configurations become energetically more favoured. Looking at spatial slices in the phase $\kappa \leq 1$, a typical two-geometry will consist of many smaller universes connected to each other by cutoff-size wormholes.

\footnotetext{
${ }^{15}$ We cannot be more specific about the nature of the interactions before having analyzed the transfer matrix of the model in more detail.

${ }^{16}$ At least, we have so far not seen any evidence of this phase or the associated second-order transition in the computer simulations.
} 


\section{The general ABAB-matrix model}

While the phase structure of the matrix model of (generalized) 3d Lorentzian gravity can be understood from the KZ-solution for the special case $\alpha_{1}=\alpha_{2}$ of the $A B A B$ matrix model, the construction of the quantum Hamiltonian requires that we perturb away from $\alpha_{1}=\alpha_{2}$. It is not difficult to generalize the ansatz of [20] to this situation, using the techniques developed in 41. One can write down a set of singular integral equations which generalize those solved in [20]. Furthermore, using results from the general theory of singular integral equations one can prove that the solution is unique.

Although we have at this stage nothing much to say about the explicit solution for general $\alpha_{1}$ and $\alpha_{2}$, it may be worth pointing out a connection with yet another $2 \mathrm{~d}$ statistical model. As mentioned in [20] and studied in more detail in [42, the $A B A B$-matrix model with $\alpha_{1}=\alpha_{2}$ can be mapped to an eight-vertex model defined on a random four-valent lattice (in the dual picture). The map is simply given by $X=A+i B$. Using the same transformation, one can map the general $A B A B$-model to a sixteen-vertex model with partition function

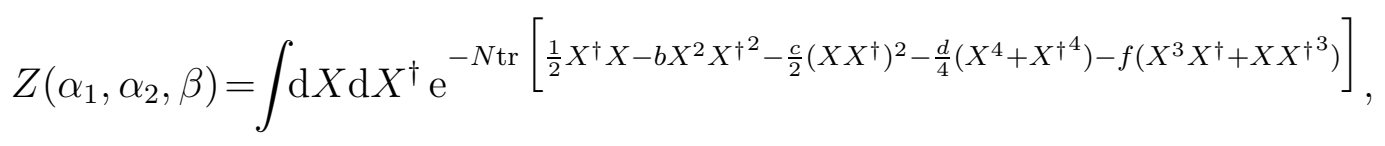

where the constants $b, c, d$, and $f$ are given by

$$
b=\frac{\alpha_{1}+\alpha_{2}+2 \beta}{16}, \quad c=d=\frac{\alpha_{1}+\alpha_{2}-2 \beta}{16}, \quad f=\frac{\alpha_{1}-\alpha_{2}}{16} .
$$

Since the eight-vertex model on a regular square lattice has already been solved, it may not be very surprising that the restricted $A B A B$-model can be solved too. This would be in line with the general observation that it is often simpler to solve matter models on random dynamical lattices (associated with $2 \mathrm{~d}$ quantum gravity) rather than on regular ones. By the same token, one should maybe not be too discouraged by the fact that the general sixteen-vertex model has not been solved on a regular lattice. This is also not what is needed here, because of the particular form of the parameters (46).

\section{Taking the continuum limit}

We have already described in some detail in Subsection 6.3 how the phase structure of the matrix model with $A B A B$-interaction can be reinterpreted from the point of view of three-dimensional geometry, and how this fits into our previous investigations of three-dimensional Lorentzian quantum gravity. One further point we would like to make concerns the nature of the continuum limit in the gravitational model, and what the newly established relation with the matrix model can add to our understanding of it. 
In dynamically triangulated models of quantum gravity one usually performs the continuum limit by fixing the bare (inverse) gravitational coupling constant $\tilde{k}$ and fine-tuning the bare cosmological constant $\tilde{\lambda}$ to its critical value $\tilde{\lambda}_{c}(\tilde{k})$. Since the space-time volume is conjugate to the cosmological constant in the action, such a fine-tuning corresponds to taking the lattice volume $N_{t o t}=N_{14}+N_{41}+\frac{1}{2} N_{22}$ to infinity, and can be viewed as an additive renormalization

$$
\tilde{\lambda}=\tilde{\lambda}_{c}(\tilde{k})+\Delta \tilde{\lambda}, \quad \Delta \tilde{\lambda}=\Lambda_{3} a^{\nu},
$$

of the cosmological constant, where $\Delta \tilde{\lambda}$ should be related to the continuum cosmological coupling constant $\Lambda_{3}$ by a suitable scaling.

The analogue of this procedure in a lattice field theory, for example, the Ising model defined on a hypercubic $N^{d}$-lattice, would be to take the infinite-lattice limit. However, this may not lead to a continuum limit. In the case of the Ising model, in order to obtain such a limit, the coupling constant $\beta$ (the inverse temperature) must always be fine-tuned to a critical value, even if the lattice volume was infinite. By analogy, one might therefore expect that also in simplicial gravity models, the gravitational coupling $\tilde{k}$ had to be fine-tuned to a critical value $\tilde{k}_{c}$ to arrive at an interesting continuum theory.

However, this is not the only possible scenario. Consider another hypercubic lattice model in $d$ dimensions, one with a lattice scalar field $\phi_{n}$ and the simple Gaussian action

$$
S[\phi]=\sum_{n} \sum_{i=1}^{d}\left(\phi_{n+\hat{e}_{i}}-\phi_{n}\right)^{2} .
$$

For this model, one automatically obtains the continuum Gaussian field theory by taking the limit of infinite lattice volume and rescaling the lattice spacing to zero. The same situation is encountered in two-dimensional simplical quantum gravity, both for Lorentzian and Euclidean signature, when formulated as a sum over triangulations with geodesic edge lengths determined by a single lattice spacing $a$. Fine-tuning the cosmological coupling constant $\tilde{\lambda}^{(2)}$ to its critical value according to

$$
\tilde{\lambda}^{(2)}=\tilde{\lambda}_{c}^{(2)}+\Delta \tilde{\lambda}^{(2)}, \quad \Delta \tilde{\lambda}^{(2)}=\Lambda_{2} a^{2},
$$

or, equivalently, taking the infinite-volume limit and scaling $a$ to zero, leads automatically to a continuum theory of $2 \mathrm{~d}$ quantum gravity. In (49), $\Lambda_{2}$ denotes the continuum two-dimensional cosmological constant, which has already appeared in our earlier discussion of Lorentzian 2d gravity (see eq. (18)).

It is easy to see that the taking of the continuum limit in the two-matrix model with $A B A B$-interaction falls into this latter category. According to [20], the continuum limit is obtained by fixing the ratio $\kappa=\alpha / \beta$ (corresponding to straight lines through the origin in Fig. (6), and increasing $\alpha$ and $\beta$ until the critical point is reached. This is a natural procedure to adopt, since the ratio $\alpha / \beta$ appears in the equations which determine the solution of the model. However, one could equally well approach the critical curve $\alpha_{c}(\kappa)=\kappa \beta_{c}(\kappa)$ along any family of curves in the 
$(\beta, \alpha)$-plane which intersect this line. In particular, one could choose curves of constant $\tilde{k}$, which according to (45) are defined by $\alpha=\tilde{k} \beta^{2}$ (c.f. Fig. (6). The curve for a given value $\tilde{k}$ intersects the critical line in the point $\left(\beta_{c}(\kappa(\tilde{k})), \alpha_{c}(\kappa(\tilde{k}))\right)$. Moving along the constant- $\tilde{k}$ curve to the critical point is of course tantamount to fine-tuning the cosmological constant, described by eq. (47).

This observation lends further support to our interpretation of the role of the gravitational coupling constant $\tilde{k}$ in $3 \mathrm{~d}$ Lorentzian quantum gravity. Based on the results of our computer simulations we suggested in [18] that the continuum limit of this theory does not involve a renormalization of $\tilde{k}$. Rather, $\tilde{k}$ should act as a finite overall scale parameter. Now, as we have seen in Sec. 6, it is natural to identify the Lorentzian model of [15, 16] with the $\kappa>1$ phase of the $A B A B$-matrix model. Forgetting for a moment about the new three-dimensional interpretation we have given it, the matrix model by itself is simply a two-dimensional statistical system with two phases and a transition point in between. In one of the phases, the scaling behaviour of the subgraphs of $A$ - or $B$-type corresponds to that found in ordinary $2 \mathrm{~d}$ Euclidean quantum gravity with $c=0$. The other phase corresponds also to $c=0$ quantum gravity, but with the "unconventional" branch of the KPZ equation chosen in the way described in subsection 6.2. Changing $\kappa$ (that is, changing $\tilde{k}$ ), but staying within the same phase, the continuum limit is of course identical, which means that the theories can differ at most by the choice of an overall length scale.

\section{Outlook}

As we have already mentioned earlier, extracting further physical information about $3 \mathrm{~d}$ Lorentzian gravity, with or without wormholes, requires a more detailed understanding of the transfer matrix (or, equivalently, of the quantum Hamiltonian). This should help us to determine the appropriate renormalization of the boundary cos-

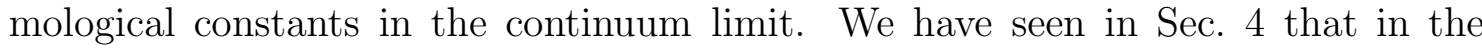
two-dimensional Lorentzian model, where the transfer matrix, the Hamiltonian and their spectra are known explicitly, the analogous scaling is canonical.

Another interesting question is how the presence of the extended three-dimensional structures observed in simulations of the Lorentzian model is reflected in the mathematical properties of the transfer matrix. We found in [18] that when the

extension in time-direction of the simulated three-geometries exceeds $\Lambda_{3}^{-1 / 3}$ - with the renormalized cosmological constant $\Lambda_{3}$ setting an effective volume scale of the system - a universe with a finite spatial volume exists only for a time duration $T \sim \Lambda^{-1 / 3}$, whereas during the remaining time the size of the (compact) spatial directions remains at the cutoff scale.

Our interpretation of the $A B A B$-matrix model raises the interesting issue of whether the phase transition of this model can be given a meaning in terms of three-dimensional geometry too. The intriguing fact that a two-dimensional central charge $c=1$ is associated with this point brings to mind attempts of associating 
$2 \mathrm{~d}$ conformal field theories with two-dimensional (boundary) surfaces in theories of 3d gravity. Examples are given by the recent entropy calculations in anti-de Sitter 433 and de Sitter space 444. We cannot directly compare with any of these results, because our set-up is quite different. We are working in a geometric and not a gauge-theoretic Chern-Simons formulation, our boundary surfaces are compact and space-like, and our (bare) cosmological coupling constant is by necessity positive. Nevertheless, the $A B A B$-matrix model is suggestive of the presence of additional symmetries at the phase transition point.

As for the topology of our model, we have restricted ourselves to discussing spacetimes $S^{2} \times[0,1]$, since the solution of the $A B A B$-matrix model as presented in [20] is valid only for spherical topology. In order to make a comparison with other approaches to $(2+1)$ quantum gravity, it would be desirable to consider also surfaces of higher genus $g$, where the physical configuration space is described by a finite number of Teichmüller parameters. Results obtained so far for the matrix model suggest that its phase structure does not change for higher genus; the values of the critical coupling constants are independent of $g$, and most critical exponents are unaltered since they refer only to the short-distance behaviour of the model. Nevertheless it will be difficult to compare with a canonical reduced phase space quantization, say, because in the matrix model all modular parameters appear integrated over for a given genus. It is possible one could find a way to probe the individual Teichmüller parameters, although it seems already quite complicated to perform the necessary large- $M$ expansion of the $A B A B$-matrix model using the character expansion of [20].

We have argued in this article that under certain assumptions the two-matrix model with $A B A B$-interaction describes three-dimensional Lorentzian quantum gravity. In fact, not only does it describe the "regular" Lorentzian quantum gravity formulated in 15, 18, but a more general theory where space can split into many components connected by "wormholes", resulting in a tree structure of spatial universes forming a branched polymer. Starting at vanishing bare Newton's constant $G_{N}$, by increasing $G_{N}$ gradually we first find a phase of "weak gravity" which seems to coincide with the phase of regular Lorentzian gravity seen previously in computer simulations. Eventually, we meet a second-order transition point beyond which lies a phase of "strong gravity" and large $G_{N}$. From our original point of view of using causality as an effective regulator of quantum geometry, it is of course unclear to what extent the space-times with an abundance of spatial wormholes found in the strong-gravity phase are still acceptable in the path integral. In order to decide this question, we need to get a better understanding of the genuinely three-dimensional properties of this system of quantum geometry. 


\section{Acknowledgements}

All authors acknowledge support by the EU network on "Discrete Random Geometry", grant HPRN-CT-1999-00161, and by ESF network no.82 on "Geometry and Disorder". In addition, J.A. and J.J. were supported by "MaPhySto", the Center of Mathematical Physics and Stochastics, financed by the National Danish Research Foundation, and J.J. by KBN grant 2P03B 01917.

\section{Appendix}

In this appendix we will calculate the Regge version of the Euclidean gravitational action (21), which we use in Sec. 2. It should be remembered that our geometries are Wick-rotated versions of discrete Lorentzian (2+1)-dimensional space-times [16]. For a change, we will do the angle calculations for Euclidean signature, and rotate back afterwards. Let the ratio of the squared lengths of time- and space-like links be given by a constant $\beta>\frac{1}{2}$, such that $l_{t}^{2}=\beta l_{s}^{2}$. The dihedral angles of $(4,1)-$ and $(1,4)$-pyramids around space- and time-like links will be denoted by $\theta_{s}$ and $\theta_{t}$ respectively. Calling the corresponding dihedral angles of the $(2,2)$-tetrahedra $\phi_{s}$ and $\phi_{t}$, one derives the relations

$$
\cos ^{2} \theta_{s}=-\cos \theta_{t}=\frac{1}{4 \beta-1}, \quad \phi_{s}=\pi-2 \theta_{s}, \quad \phi_{t}=\pi-\theta_{t}
$$

The numbers of the three different types of building blocks filling out the spacetime between $t$ and $t+1$ are $N_{14}(t), N_{41}(t)$ and $N_{22}(t)$. From this we compute the number of time-like links between $t$ and $t+1$ as $N_{1}^{\mathrm{TL}}(t)=N_{14}(t)+N_{41}(t)+N_{22}(t)+2$. The number of spatial links contained in the spatial slice at $t$ is $2 N_{41}(t)$, and each spatial link at $t$ belongs to two $(4,1)$-pyramids. Collecting all this information and using the well-known expressions for Regge curvature in terms of dihedral angles [45], including boundary terms [46], the curvature contribution to the total action is found to be

$$
\begin{aligned}
& \frac{1}{2} \int_{M} \mathrm{~d}^{3} x \sqrt{g} R(x)+\int_{\partial M} \mathrm{~d}^{2} x \sqrt{h} K(x) \longrightarrow \\
& \sum_{t} a\left(4 \pi \sqrt{\beta}+\left[\left(2 \pi-4 \theta_{t}\right) \sqrt{\beta}+\left(2 \pi-4 \theta_{s}\right)\right]\left(N_{41}(t)+N_{14}(t)-N_{22}(t)\right)\right)
\end{aligned}
$$

where the term in square brackets is always positive. The total volume contributing to the cosmological term in the action is given by

$$
\int_{M} \mathrm{~d}^{3} x \sqrt{g} \longrightarrow \frac{a^{3} \sqrt{\beta-\frac{1}{2}}}{3} \sum_{t}\left(N_{41}(t)+N_{14}(t)+\frac{1}{2} N_{22}(t)\right) .
$$

\footnotetext{
${ }^{17}$ The constants $\alpha$ and $\beta$ used in this appendix have nothing to do with the couplings $\alpha$ and $\beta$ appearing in the main text.
} 
Putting in the appropriate (bare) coupling constants, this leads to a total discretized action

$$
S[\tilde{k}, \tilde{\lambda}]=c_{0} t-\tilde{k}\left(N_{41}+N_{14}-N_{22}\right)+\tilde{\lambda}\left(N_{41}+N_{14}+\frac{1}{2} N_{22}\right)
$$

where now the total numbers of the different building blocks appear and where the constants are given by

$$
\begin{gathered}
\tilde{k}=\frac{a}{4 \pi G_{N}}\left(-\pi \sqrt{\beta}+2 \sqrt{\beta} \arccos \frac{1}{4 \beta-1}+\arcsin \frac{\sqrt{\beta-\frac{1}{2}}}{\beta-\frac{1}{4}}\right), \\
\tilde{\lambda}=\frac{a^{3} \Lambda}{24 \pi G_{N}} \sqrt{\beta-\frac{1}{2}}, \quad c_{0}=-\frac{a \sqrt{\beta}}{2 G_{N}} .
\end{gathered}
$$

In order to obtain the Lorentzian version of the action (53) (up to an imaginary factor $-i$ ), we need to replace $\beta \rightarrow-\alpha$ such that $l_{t}^{2}=-\alpha l_{s}^{2}$, in accordance with the notation in [15, 16]. To get the correct Lorentzian action, one then inverts the prescription given in [15, 16] by continuing the square-root expressions according to $\sqrt{\beta} \rightarrow i \sqrt{\alpha}$ and $\sqrt{\beta-\frac{1}{2}} \rightarrow i \sqrt{\alpha+\frac{1}{2}}$.

\section{References}

[1] V.G. Knizhnik, A.M. Polyakov and A.B. Zamolodchikov: Fractal structure of 2d-quantum gravity, Mod. Phys. Lett. A3 (1988) 819.

[2] J. Distler and H. Kawai: Conformal field theory and 2-D quantum gravity, Nucl. Phys. B 321 (1989) 509;

F. David: Conformal field theories coupled to 2-d gravity in the conformal gauge, Mod. Phys. Lett. A 3 (1988) 1651.

[3] H. Kawai, N. Kawamoto, T. Mogami and Y. Watabiki: Transfer matrix formalism for two-dimensional quantum gravity and fractal structures of space-time, Phys. Lett. B 306 (1993) 19-26 hep-th/9302133.

[4] J. Ambjørn and Y. Watabiki: Scaling in quantum gravity, Nucl. Phys. B 445 (1995) 129-144 hep-th/9501049.

[5] J. Ambjørn, J. Jurkiewicz and Y. Watabiki: On the fractal structure of two-dimensional quantum gravity, Nucl. Phys. B 454 (1995) 313-342 [heplat/9507014].

[6] J. Ambjørn and R. Loll: Non-perturbative Lorentzian quantum gravity, causality and topology change, Nucl. Phys. B 536 (1998) 407-434 [hep-th/9805108].

[7] J. Ambjørn, K.N. Anagnostopoulos and R. Loll: A new perspective on matter coupling in 2d quantum gravity, Phys. Rev. D 60 (1999) 104035 hepth/9904012. 
[8] J. Ambjørn, K.N. Anagnostopoulos and R. Loll: Crossing the $c=1$ barrier in 2d Lorentzian quantum gravity, Phys. Rev. D 61 (2000) 044010 [hep-lat/9909129].

[9] J. Ambjørn, J. Correia, C. Kristjansen and R. Loll: The relation between Euclidean and Lorentzian 2D quantum gravity, Phys. Lett. B 475 (2000) 24-32 hep-th/9912267.

[10] S. Catterall, G. Thorleifsson, M. Bowick and V. John: Scaling and the fractal geometry of two-dimensional quantum gravity, Phys. Lett. B 354 (1995) 58-68 [hep-lat/9504009.

[11] J. Ambjørn and J. Jurkiewicz: Four-dimensional simplicial quantum gravity, Phys. Lett. B 278 (1992) 42-50.

[12] M.E. Agishtein and A.A. Migdal: Simulations of four-dimensional simplicial quantum gravity, Mod. Phys. Lett. A 7 (1992) 1039-1061.

[13] P. Bialas, Z. Burda, A. Krzywicki and B. Petersson: Focusing on the fixed point of 4-d simplicial gravity., Nucl. Phys. B 472 (1996) 293-308 hep-lat/9601024.

[14] R. Loll: Discrete approaches to quantum gravity in four dimensions, Living Rev. Rel. 1, 13 (1998), http://www.livingreviews.org [gr-qc/9805049].

[15] J. Ambjørn, J. Jurkiewicz and R. Loll: A nonperturbative Lorentzian path integral for gravity, Phys. Rev. Lett. 85 (2000) 924-927 hep-th/0002050.

[16] J. Ambjørn, J. Jurkiewicz and R. Loll: Dynamically triangulating Lorentzian quantum gravity, preprint Golm AEI-2001-049 hep-th/0105267.

[17] R. Loll: Discrete Lorentzian quantum gravity, Nucl. Phys. B (Proc. Suppl.) 94 (2001) 96-107 [hep-th/0011194].

[18] J. Ambjørn, J. Jurkiewicz and R. Loll: Nonperturbative 3d Lorentzian quantum gravity., to appear in Phys. Rev. D [hep-th/0011276.

[19] J. Ambjørn, J. Jurkiewicz and R. Loll: Computer simulations of 3d Lorentzian quantum gravity, Nucl. Phys. B (Proc. Suppl.) 94 (2001) 689-692 heplat/0011055.

[20] V.A. Kazakov and P. Zinn-Justin: Two matrix model with ABAB interaction, Nucl. Phys. B 546 (1999) 647-668 hep-th/9808043].

[21] Y. Watabiki: Construction of noncritical string field theory by transfer matrix formalism in dynamical triangulation, Nucl. Phys. B 441 (1995) 119-166 hepth/9401096]. 
[22] H. Aoki, H. Kawai, J. Nishimura and A. Tsuchiya: Operator product expansion in two-dimensional quantum gravity, Nucl. Phys. B 474 (1996) 512-528 [hepth/9511117].

[23] A. Dasgupta and R. Loll: A proper-time cure for the conformal sickness in quantum gravity, Nucl. Phys. B, to appear [hep-th/0103186].

[24] E. Martinec: Soluble systems in quantum gravity, Phys. Rev. D 30 (1984) 11981204.

[25] A. Hosoya and K. Nakao: (2+1)-dimensional quantum gravity, Prog. Theor. Phys. 84 (1990) 739-748.

[26] P. Di Francesco, E. Guitter and C. Kristjansen: Integrable 2-d Lorentzian gravity and random walks, Nucl. Phys. B 567 (2000) 515-553 [hep-th/9907084; Generalized Lorentzian gravity and the Calogero Hamiltonian, preprint SACLAYSPHT-T00-145 hep-th/0010259.

[27] S.R. Das, A. Dhar, A.M. Sengupta and S.R. Wadia: New critical behaviour in $d=0$ large- $N$ matrix models, Mod. Phys. Lett. A 5 (1990) 1041-1056.

[28] G.P. Korchemsky: Loops in the curvature matrix model, Phys. Lett. B 296 (1992) 323-334 hep-th/9206088.

[29] L. Chekhov and C. Kristjansen: Hermitian matrix model with plaquette interaction, Nucl. Phys. B 479 (1996) 683-696 hep-th/9605013.

[30] I.K. Kostov: $O(N)$ vector model on a planar random lattice: spectrum of anomalous dimensions, Mod. Phys. Lett. A 4 (1989) 217.

[31] B. Duplantier and I. Kostov: Conformal spectra of polymers on a random surface, Phys. Rev. Lett. 61 (1988) 1433.

[32] I.K. Kostov and M. Staudacher: Multicritical phases of the $O(n)$ model on a random lattice, Nucl. Phys. B 384 (1992) 459-483 hep-th/9203030.

[33] J. Ambjørn, B. Durhuus and J. Fröhlich: The appearance of critical dimensions in regulated string theories (II), Nucl. Phys. B 275 (1986) 161.

[34] B. Durhuus: Multi-spin systems on a randomly triangulated surface, Nucl. Phys. B 426 (1994) 203-222 hep-th/9402052.

[35] J. Ambjørn, B. Durhuus and T. Jonsson: A solvable 2-d gravity model with $\gamma>0$, Mod. Phys. Lett. A 9 (1994) 1221-1228 hep-th/9401137.

[36] I.R. Klebanov: Touching random surfaces and Liouville gravity, Phys. Rev. D 51 (1995) 1836-1841 hep-th/9407167. 
[37] I.R. Klebanov and A. Hashimoto: Nonperturbative solution of matrix models modified by trace squared terms, Nucl. Phys. B 434 (1995) 264-282 [hepth/9409064.

[38] J.L.F. Barbon, K. Demeterfi, I.R. Klebanov and C. Schmidhuber: Correlation functions in matrix models modified by wormhole terms, Nucl. Phys. B 440 (1995) 189-214 hep-th/9501058.

[39] T. Jonsson and J.F. Wheater: The spectral dimension of the branched polymer phase of two-dimensional quantum gravity, Nucl. Phys. B 515 (1998) 549-574 hep-lat/9710024.

[40] J. Ambjørn, D. Boulatov, J.L. Nielsen, J. Rolf and Y. Watabiki: The spectral dimension of 2-d quantum gravity, JHEP 9802:010 (1998) hep-th/9801099.

[41] V.A. Kazakov, M. Staudacher and T. Wynter: Exact solution of discrete twodimensional $R^{2}$ gravity, Nucl. Phys. B 471, (1996) 309-333 hep-th/9601069;

Almost flat planar diagrams, Commun. Math. Phys. 179 (1996) 235-256 [hepth/9506174]; Character expansion methods for matrix models of dually weighted graphs, Commun. Math. Phys. 177 (1996) 451-468 [hep-th/9502132;

P. Zinn-Justin, Random hermitian matrices in an external field, Nucl. Phys. B 497 (1997) 725-732 cond-mat/9703033.

[42] P. Zinn-Justin: The six-vertex model on random lattices, Europhys. Lett. 50 (2000) 15-21 cond-mat/9909250.

[43] S. Carlip: Statistical mechanics of the (2+1)-dimensional black hole, Class. Quant. Grav. 12 (1995) 2853-2879 gr-qc/9409052;

O. Coussaert, M. Henneaux and P. van Driel: The asymptotic dynamics of three-dimensional Einstein gravity with a negative cosmological constant, Class. Quant. Grav. 12 (1995) 2961-2966 gr-qc/9506019.

[44] J. Maldacena and A. Strominger: Statistical entropy of de Sitter space, JHEP 9802:014 (1998) gr-qc/9801096;

M. Bañados, T. Brotz and M.E. Ortiz: Quantum three-dimensional de Sitter space, Phys. Rev. D 59 (1999) 046002 hep-th/9807216.

[45] T. Regge: General relativity without coordinates, Nuovo Cim. A 19 (1961) 558571. [hep-th/9807216.

[46] J.B. Hartle and R. Sorkin: Boundary terms in the action for the Regge calculus, Gen. Rel. Grav. 13 (1981) 541-549. 